\title{
Comparison of Quantitative and Qualitative Information Provided by Different Structural Load Path Definitions
}

\author{
Kun Marhadi $^{1}$, Satchi Venkataraman ${ }^{2, a}$ \\ ${ }^{1}$ Computational Science Research Center, San Diego State University, San Diego, United States \\ ${ }^{2}$ Department of Aerospace Engineering and Engineering Mechanics, San Diego State University, San Diego, United States
}

Received 5 June 2009, Accepted 17 August 2009

\begin{abstract}
The structural mechanics community has developed several methods to identify and visualize load paths in structures. There is no single accepted approach or method to characterize, quantify, visualize and tailor load paths. This paper compares various methods proposed to characterize and visualize load paths. The comparison is performed for a twodimensional (2-D) rectangular plate under plane stress conditions arising from different loading and boundary conditions. The effectiveness of each method is compared. The comparisons shows the methods provide different results and insights into the load transfer mechanisms. No single method appears to emerge as a clear choice for characterizing load paths. However, some methods provide more qualitative and quantitative information on the trajectory and efficiency of load transferred along load paths.
\end{abstract}

Key words: Structural load path definitions, Alternate load paths

\section{Introduction}

Structural design engineers need to be able to visualize how a load is transferred from its application point to the support or reaction points. Knowing how load "flows" in a structure is important at the design stage to make sure that a structure will perform its intended function properly. It is also important for optimum material utilization and for assessing the overall integrity of a structure. In case the structure is damaged, it is crucial to anticipate how the load flow will change in order to ensure that the structure can continue to perform its basic functions. Thus, it is important to identify alternate load paths in case the primary load path is damaged. In addition to identifying alternate load paths, engineers may want to be able to specifically tailor alternate load paths so that the structure can perform its basic functions under various unforeseen damage conditions.

Although it is clear why knowledge of load pathways is important, no commonly accepted approach has been developed to quantify, characterize, and visualize available load paths in a structure. Ideally, a method that visualizes load paths in a structure should be able to (1) visualize the overall paths of forces from the points of loading application to the reactions points, (2) indicate regions that need stiffness tailoring (more or less material utilization), and (3) indicate critical regions (regions with high stresses). The first criterion will help determine whether the structure carries loads as intended in the design. The second criterion provides information for optimum material utilization, and the last criterion helps anticipate regions with failure possibility. This work presents and compares different methods that have been developed for characterizing load paths. The methods are used to visualize load paths in a rectangular plate structure under various boundaries and loading conditions.
Condition in which a hole exists in the structure (stiffness changes) is also considered. For each condition, the interpretation that can be obtained from visualization of each method is analyzed and checked whether it can meet criteria defined earlier.

\section{Load Path Definitions}

Over the years, the structural community has developed several methodologies that can be used to define load paths in a structure. Each method was derived differently, and therefore results in varied visualizations and interpretations of structural load paths. The different methods investigated in this work are explained in the following sections.

\subsection{Using vectors aligned with directions of principal stresses}

Principal stress vectors are readily available from postprocessing program available in most commercial finite element analyses. As a result, the trajectory of principal direction vectors is interpreted as load paths. From theory of elasticity [1], in plane normal stress $\sigma_{\mathrm{n}}$ and shearing stress $\tau_{\mathrm{nt}}$ acting at a point on an arbitrary plane of orientation angle $\theta$ with $x$ axis stress equations are defined by Equations 1 and 2. The stresses are computed in terms of stresses: $\sigma_{x}, \sigma_{y}$, and $\tau_{\mathrm{xy}}=\tau_{\mathrm{yx}}$ along the coordinate axes as shown in Figure 1.

$$
\begin{gathered}
\sigma_{n}=\sigma_{x} \cos ^{2} \theta+\sigma_{y} \sin ^{2} \theta+\tau_{x y} \sin \theta \cos \theta \\
\tau_{n t}=-\left(\sigma_{x}-\sigma_{y}\right) \sin \theta \cos \theta+\tau_{x y}\left(\cos ^{2} \theta-\sin ^{2} \theta\right)
\end{gathered}
$$

The principal stress directions $\left(\theta_{p}\right)$ obtained by setting equation 2 equal to zero provides the principal direction,

\footnotetext{
a Corresponding author: satchi@mail.sdsu.edu
} 
expressed as

$$
\tan 2 \theta_{p}=\frac{2 \tau_{x y}}{\sigma_{x}-\sigma_{y}} .
$$

The angles of major $\left(\sigma_{p 1}\right)$ and minor $\left(\sigma_{p 2}\right)$ principal stress directions are $\theta_{p 1}$ and $\theta_{p 2}$ respectively. The two angles $\theta_{p 1}$ and $\theta_{p 2}$ are $90^{\circ}$ apart. The two principal stresses can be computed by replacing $\theta$ with $\theta_{p 1}$ and $\theta_{p 2}$ in Equation 1 . The major principal stress $\sigma_{\mathrm{p} 1}$ corresponds to the larger magnitude of the two stresses.
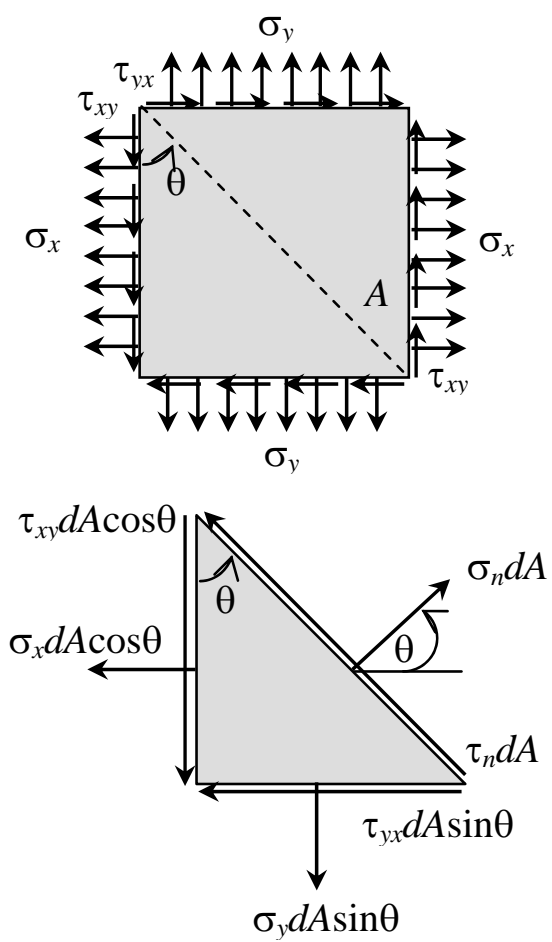

Fig 1. Geometry and notation for stress components acting on an arbitrary plane

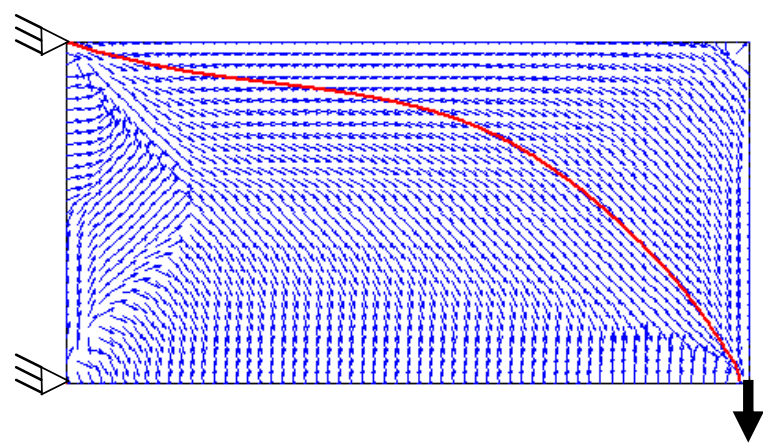

(a) Vector fields aligned with major principal stress $\sigma_{p 1}$, load trajectory (red line) is traced from upper support

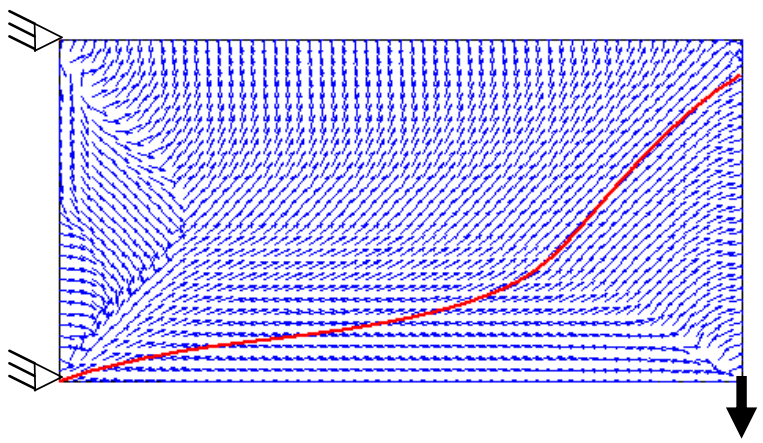

(b) Vector fields aligned with minor principal stress $\sigma_{p 2}$, load trajectory is traced from lower support

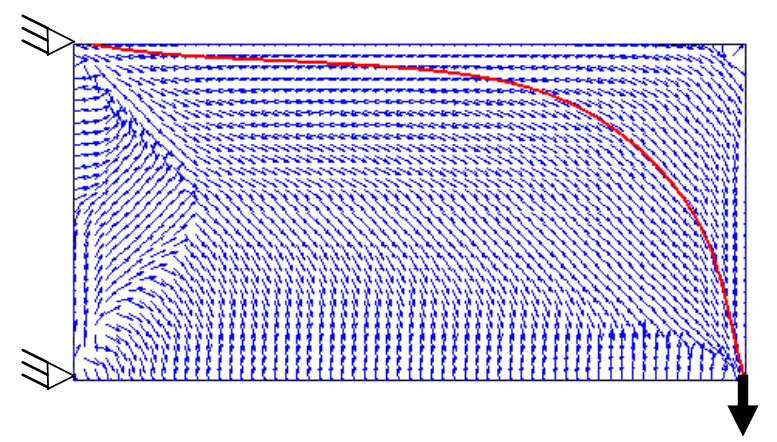

(c) Vector fields aligned with major principal stress $\sigma_{p 1}$ with load trajectory traced from the loading point

Fig. 2. A 2D homogeneous rectangular plate

Figure 2 shows an isotropic rectangular plate loaded vertically at its right bottom corner and constrained at the left corners. Stresses in the structure were computed using ABAQUSTM finite element analysis software. The rectangular plate is meshed with 30 by 60 4-noded quadrilateral plate finite elements with reduced integration. Figure 2 (a) and (b) show the principal stress $\left(\sigma_{p 1}\right.$ and $\left.\sigma_{p 2}\right)$ vectors (unit vectors) respectively at each element center in the structure. The red line indicates an attempt to trace trajectory of load following the principal direction from the reaction points to the point of loading application. This is a way to visualize paths of forces from the points of loading application to the reactions points. Tracing trajectory following the orientation of principal stresses from the point of loading application produces different results as opposed to following the trajectory from reaction points (see Figure 2 (c)). In the orientation of major principal stress, tracing trajectory from the upper support ends a location very close to the loading point. Similarly, tracing trajectory from the loading point ends at a location very close to the upper support. Ideally, trajectories that connect loading points and reaction points should be the same when they are traced from either the reaction or loading points.

In this example, the nature of loading on the structure is such that the loaded end (right side) of the plate is predominantly under shear load. However as we move towards the supports on the left, the structure develops bending and the load is resisted by a combination of shear 
and bending. By plotting orientation vectors of $\sigma_{p 1}$ and $\sigma_{p 2}$ separately, the flow of stresses in tensile $\left(\sigma_{p 1}\right)$ and in compression $\left(\sigma_{p 2}\right)$ can be visualized independently. $\sigma_{p 1}$ is maximum near the upper left support and diminish to zero at the lower side of the structure. Similarly, $\sigma_{p 2}$ is maximum near the lower left support and diminish to zero at the upper side of the structure. Since often times we are more interested in regions where the stresses (compression and tension) are high, it becomes more informative to plot the orientation vector of the maximum of the absolute value of either $\sigma_{p 1}$ or $\sigma_{p 2}\left(\max \left(\operatorname{abs}\left(\sigma_{p 1}\right), \operatorname{abs}\left(\sigma_{p 2}\right)\right)\right.$ in each element at the same plot. This way, we can visualize the flow of high stresses (or load paths) as shown in Figure 3 (a). The upper half of the structure is predominantly under tensile stress, and the lower half is predominantly under compression stress. Tracing the trajectories from both supports by following the orientation vectors ends at the loaded point. Plotting the contour of maximum absolute value of principal stresses can indicate high stress regions as shown in Figure 3 (b). The contours are relatively similar with contours of von Misses stress of the structure as shown in Figure 3 (c). Here, high stress regions are near the supports and point of loading application.

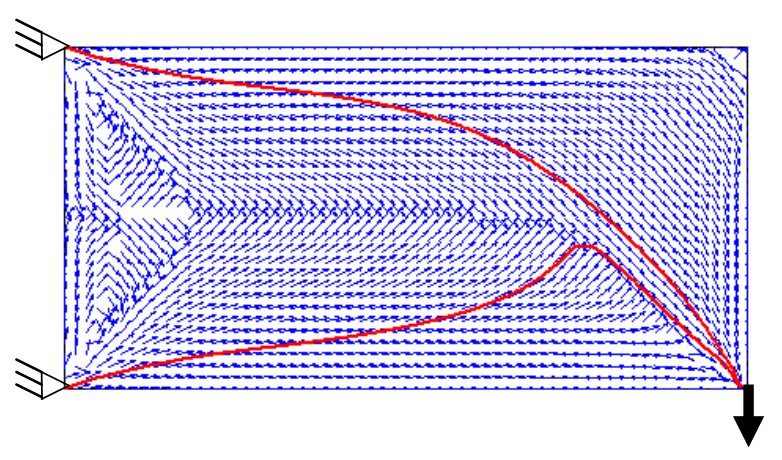

(a) Orientation vector of $\max \left(\operatorname{abs}\left(\sigma_{\mathrm{p} 1}\right), \mathrm{abs}\left(\sigma_{\mathrm{p} 2}\right)\right)$ in each element

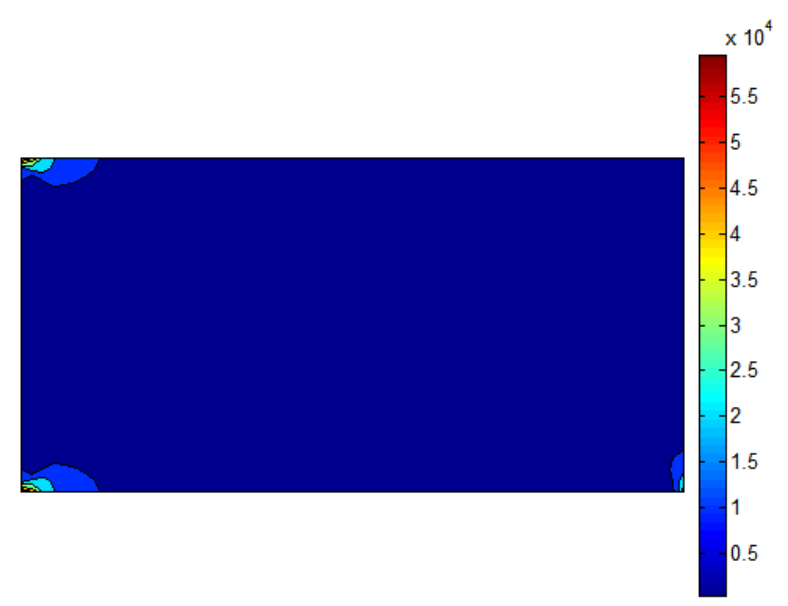

(b) Contours of magnitude of $\max \left(\operatorname{abs}\left(\sigma_{\mathrm{p} 1}\right), \operatorname{abs}\left(\sigma_{\mathrm{p} 2}\right)\right)$ in each element

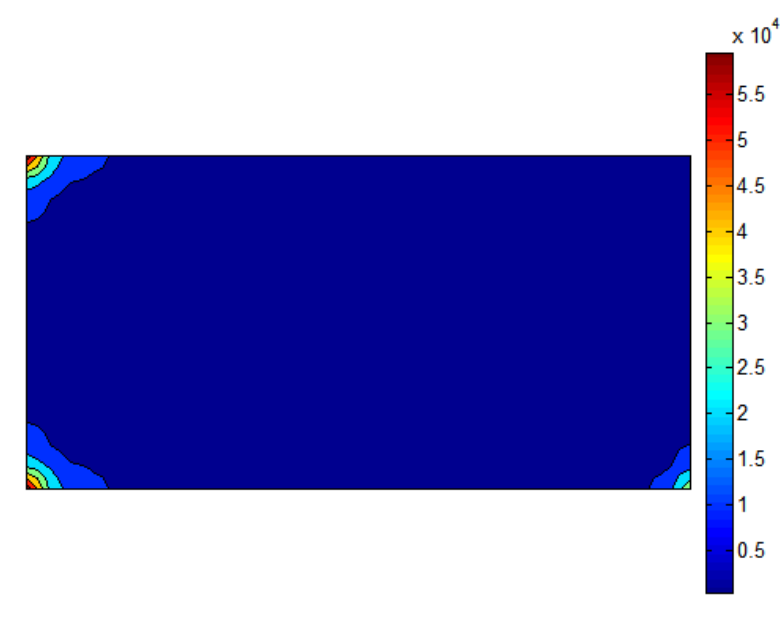

(c) Contours of von Misses stress.

Fig.3. Stresses on rectangular plate

The principal stresses and their directions indicate load distribution in the plate from the load introduction point to the support point. Kelly and Tosh [2] and Kelly et al. [3] explained that plotting orientations of the principal stresses does not indicate regions or contours where the load is constant as in a fluid flow streamline. Therefore tracing load trajectories based on these orientations will not result in trajectories where the loads being transferred are constant in the sense of streamlines in a fluid flow. To obtain trajectories that are similar to streamlines in a fluid flow, Kelly et al. [3] proposed a modified method for obtaining load trajectories from the stress field using fluid flow analogy. This is described in the next section.

\subsection{Load path based on load flow}

In the modified procedure of Kelly et al. [3], the load path is defined as the trajectory along which a constant force is being transferred. The concept is derived from fluid flow analogy. Although stress analysis does not obey the continuity law in the sense of fluid flow, continuity can be applied to the components of the force in an arbitrary set of orthogonal directions because of equilibrium requirements. This concept is illustrated with a hypothetical force "stream tube”, shown in Figure 4.

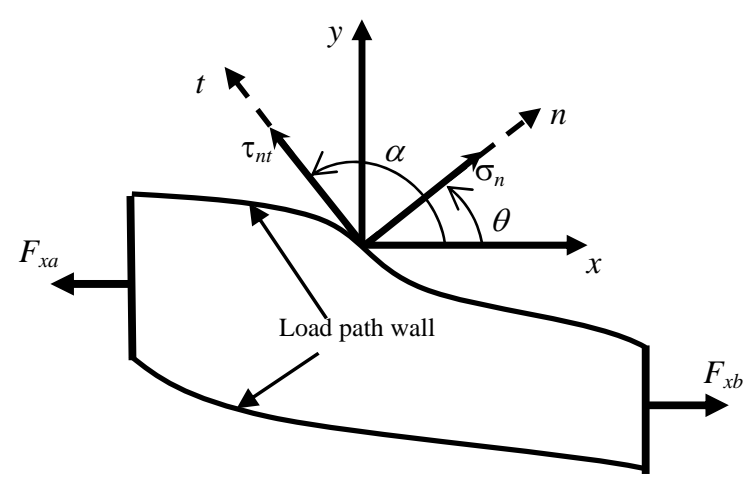


Fig. 4. Schematic of force "stream tube" that must satisfy equilibrium

Waldman et al. [4] summarized the theory originally derived by Kelly and Elsley [5] with some further clarification. They explained that for a particular force direction, such as the $x$-direction depicted in Figure 4, there is no flow of force across the boundary of the force tube. The load path is bounded by lines where there are no contributions to the force in the $x$-direction. Equilibrium over the tube that must be satisfied in the chosen direction (e.g. $x$-direction) can be expressed as

$$
F_{x a}-F_{x b}=0 \text {. }
$$

There are two stress components acting on a plane that is tangent to the wall of the force tube, namely $\sigma_{n}$ and $\tau_{n t}$. $\sigma_{n}$ is a local stress acting perpendicular to the plane, and $\tau_{n t}$ is acting along the plane (shear stress). The direction of $\sigma_{n}$ is oriented by an angle $\theta$ with respect to $x$-axis in Figure 4 . In this example, the load path is determined by resolving force equilibrium in the horizontal direction ( $x$-axis). Similarly, the load path can be determined by resolving force equilibrium in the vertical direction ( $y$-axis).

Equilibrium in any segment along the load path wall is achieved when the components of $\sigma_{n}$ and $\tau_{n t}$ in the $x$ directions cancel out. This condition is satisfied by the following equation $\left(\theta=\theta_{x}\right)$ :

$$
\sigma_{n} \cos \theta_{x}-\tau_{n t} \sin \theta_{x}=0 .
$$

When the force equilibrium is resolved in the $y$ direction, the equation is as follows $\left(\theta=\theta_{y}\right)$ :

$$
\sigma_{n} \sin \theta_{y}+\tau_{n t} \cos \theta_{y}=0 \text {. }
$$

Substituting $\sigma_{n}$ and $\tau_{n t}$ in Equations 5 and 6 with Equations 1 and 2 respectively, the orientation angles can be expressed as

$$
\begin{aligned}
& \tan \theta_{x}=-\frac{\sigma_{x}}{\tau_{x y}} \\
& \tan \theta_{\mathrm{y}}=-\frac{\tau_{x y}}{\sigma_{y}} .
\end{aligned}
$$

The local orientation of the load path along the wall of the force tube is expressed $\alpha$, which is $90^{\circ}$ apart from $\theta$ (see Figure 4). When the equilibrium is resolved into $x$ and $y$ directions, the angle can be expressed as

$$
\begin{aligned}
& \alpha_{x}=\tan ^{-1} \frac{\tau_{x y}}{\sigma_{x}} \\
& \alpha_{y}=\tan ^{-1} \frac{\sigma_{y}}{\tau_{x y}} .
\end{aligned}
$$

Figures 5 (a) and (b) show load paths or orientations of the load flow calculated using the force "stream tube" concept, for the $x$ and $y$-direction loads respectively. The arrows are unit vectors with orientation angles $\alpha_{x}$ and $\alpha_{y}$.
The red lines trace the trajectory of path obtained by following the load flow orientation vector from the support points where the load is reacted to the point of loading application. Here the trajectories, obtained using the load flow orientations connect the load to its support points for both forces with equilibrium along $x$ and $y$ directions. However, tracing the trajectories from the point of loading application produces different trajectories, such as shown in Figure 5 (c) for load flow with equilibrium in $y$-direction. For load flow with equilibrium in $x$-direction, no trajectories can be drawn when the starting point is the point of loading. In reality, the trajectory only connects the loading point and a point very closed to the upper reaction points. Similarly, when the trajectories are traced from the supports, they end at a point very closed to the loading point.

Analyzing load flow of $x$-direction forces reveals that the structure develops a bending moment due to the loading. The plots also indicate regions that have recirculation patterns (that resemble eddies in fluids) near the supports. These regions are typically low stress regions that do not contribute to loads in the specified load and support configuration. In the center line of the structure within the loop, the stresses are zero, and the vectors of load flow are perpendicular to the $x$-axis.

For load flow with equilibrium in the $y$-direction, the vectors show that loads are transferred from the point of application to the reaction points. Since the only non-zero component in the $y$-direction is shear, the vectors and trajectories indicate how shear is transferred. It is also evident from Figure 5 (b) that the regions with re-circulating patterns or eddies: one near the load application point and two near the reaction points, experience relatively low stress.

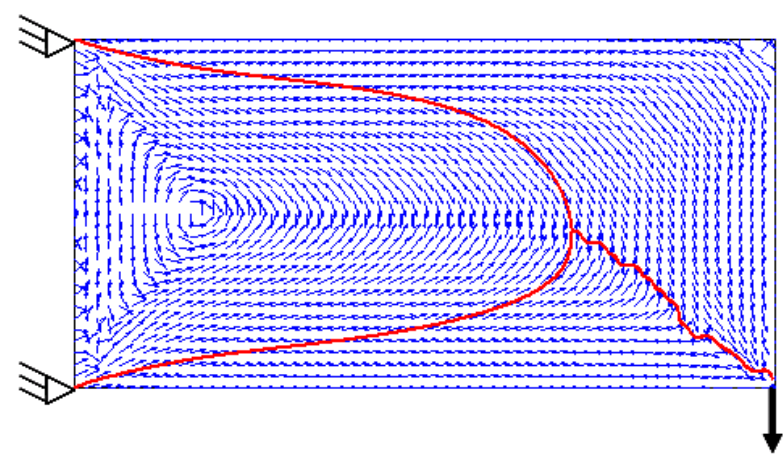

(a) $x$-direction

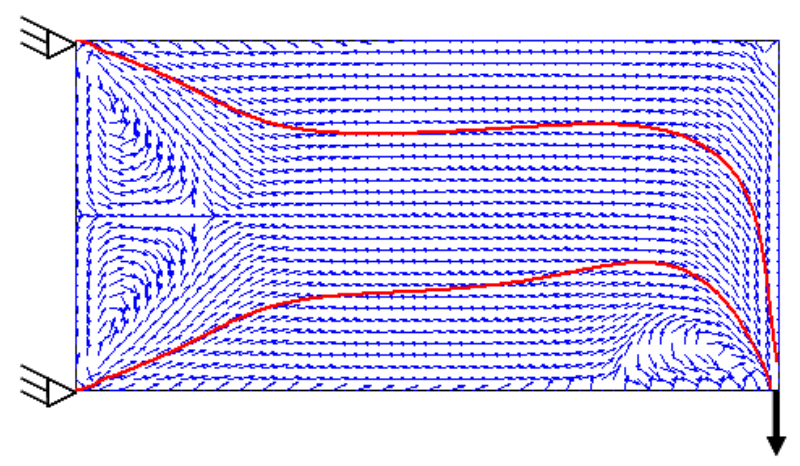

(b) $y$-direction 


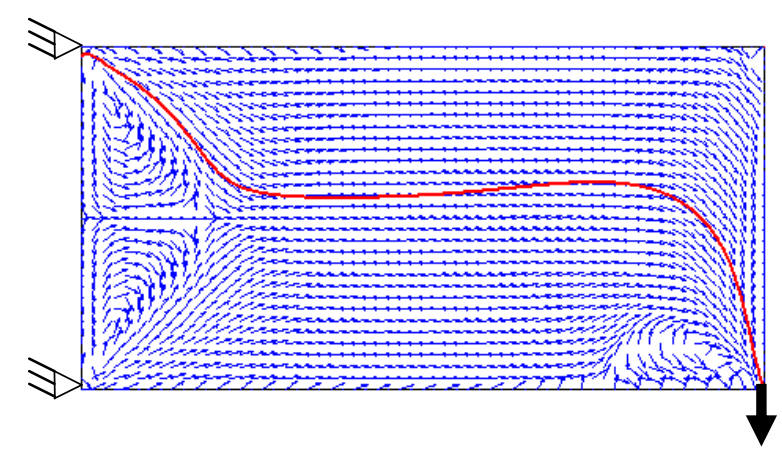

(c) Load flow orientation with force equilibrium in $y$ direction and trajectory traced from point of loading application

Fig. 5. Load flow orientation and load trajectories traced from reaction points with force equilibrium

\subsection{Transferred and potential transferred force method}

Harasaki and Arora [6] introduced the concept of transferred forces and potential transferred forces to investigate load paths in structures. Their idea is that a portion of the load is transferred through a region of the structure. The continuum representation of the structure shown in Figure 6 is used to explain this concept.

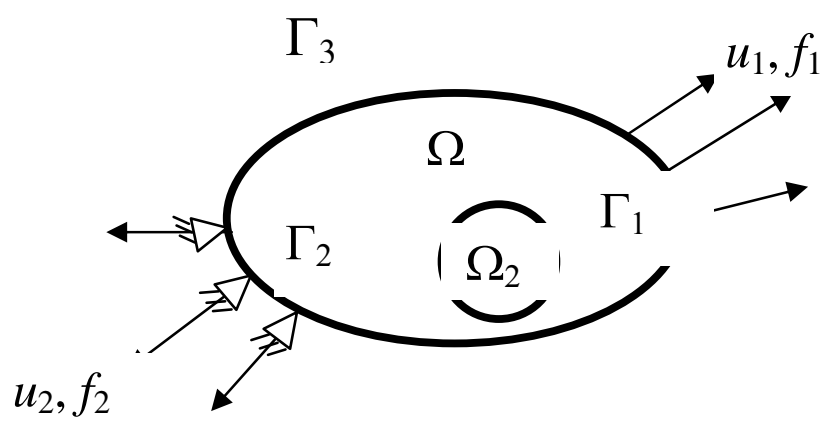

Fig. 6. Schematic representation of a continuum structure

Load $f_{1}$ applied on $\Gamma_{1}$ is transmitted to the supports on $\Gamma_{2}$ to generate reaction forces $f_{2}$. These forces can be obtained from solving the following equilibrium and boundary condition equations:

$$
\begin{aligned}
& \sigma_{i j, j}+b_{i}=0 \text { for } \Omega \text { and } \Omega_{2}, \\
& \sigma_{i j} n_{j}=f_{1} \text { on } \Gamma_{1}, \\
& u_{i}=0 \text { on } \Gamma_{2}, \\
& \sigma_{i j} n_{j}=f_{2 i} \text { on } \Gamma_{2}, \\
& \sigma_{i j} n_{j}=0 \text { on } \Gamma_{3} .
\end{aligned}
$$

The force transmitted to $\Gamma_{2}$ has two components, one that goes through region $\Omega_{2}$, and one that goes through the remaining region. The force components transferred through region $\Omega_{2}$ are referred to as the transferred force. To calculate the transferred force through $\Omega_{2}$, obtain the displacement $u$ and the reaction force $f_{2}$ for the original problem by solving Equation (11). Next, set the stiffness of region $\Omega_{2}$ to zero, apply the displacements calculated from the original solution on $\Gamma_{1}$ as enforced displacements, and obtain the new reaction load $\bar{f}_{2}$ (using the equations of equilibrium and boundary conditions shown in Equation (12)). Bar on the notations indicates a condition when stiffness of $\Omega_{2}$ is set to zero.

$$
\begin{aligned}
& \bar{\sigma}_{i j, j}+b_{i}=0 \text { for } \Omega, \\
& \bar{\sigma}_{i j}=0 \text { for } \Omega_{2}, \\
& u_{i}=u_{1 i} \text { on } \Gamma_{1}, \\
& u_{i}=0 \text { on } \Gamma_{2}, \\
& \bar{\sigma}_{i j} n_{j}=\bar{f}_{2 i} \text { on } \Gamma_{2}, \\
& \bar{\sigma}_{i j} n_{j}=0 \text { on } \Gamma_{3} .
\end{aligned}
$$

The transferred force is then defined as the difference in the reaction loads.

$$
\bar{F}=\bar{f}_{2 i}-f_{2 i} \text { on } \Gamma_{2} .
$$

The transferred force can be implemented in a finite element analysis, wherein the region $\Omega_{2}$ can be either an element of the finite element mesh or a structural member in the finite element model of the structural assembly (e.g. truss and frame structures). Harasaki and Arora [6] also introduced the concept of potential transferred forces. Potential transferred forces are forces in a region $\Omega_{2}$, in which the structure can transfer when this region is made to be rigid or to have very high value of stiffness approaching infinity. The potential transferred force gives an indication of how well stiffening the region will improve the stiffness of the structure.

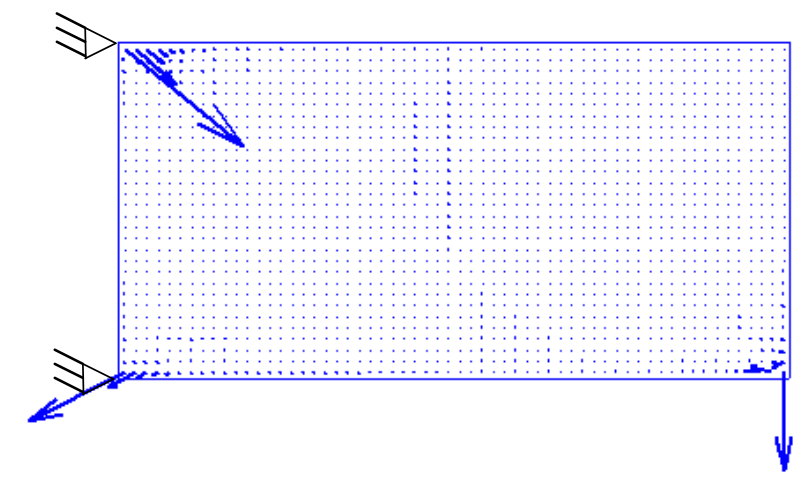

(a) Transferred force 


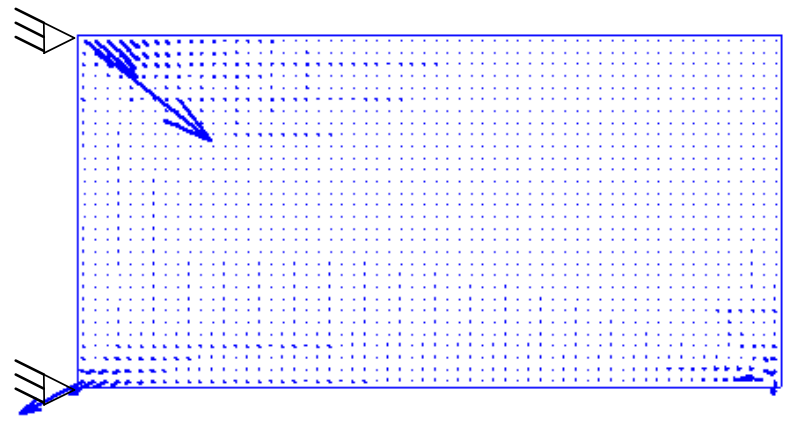

(b) Potential transferred force

Fig. 7. Transferred and potential transferred force to the reaction points

Figure 7 (a) shows an example of using transferred force method to visualize how each point on the plate contributes to the loads transfer to the support points in the homogeneous rectangular plate. Figure 7 (b) shows the potential of transferred force in the structure. The size of the arrows indicates magnitude of the transferred and potential transferred force. The transferred force and potential transferred forces plotted in Figure 7 (a) and (b) are obtained using a finite element mesh of $1800(30 \times 60)$ elements. When the structure is discretized finely, area of each element becomes smaller and thus contributes a smaller transferred force or presents a smaller potential transferred force through each element. To illustrate this point, the transferred and potential transferred forces are computed again for the structure with 50 elements (Figure 8). With coarser mesh, the contribution of each element in transferring force or its potential transfer force is larger as shown in the figure. This indicates the method is sensitive to the discretization length.

The plot of transferred force in Figure 7 (a) shows that stiffness reductions to elements near the supports and point of loading application leads to the largest changes in the load transfer (load paths). The regions with significant transferred forces are very similar with regions with high stresses (Figure 3). The plot of potential transferred force in Figure 7 (b) however shows that elements near the supports are most effective in changing the load transfer when their stiffness is increased. In subsequent papers [7] and [8], Harasaki and Arora demonstrated the attractiveness of this approach for tailoring direct stiffening or redesigning a structure such as in the case of topology optimization.

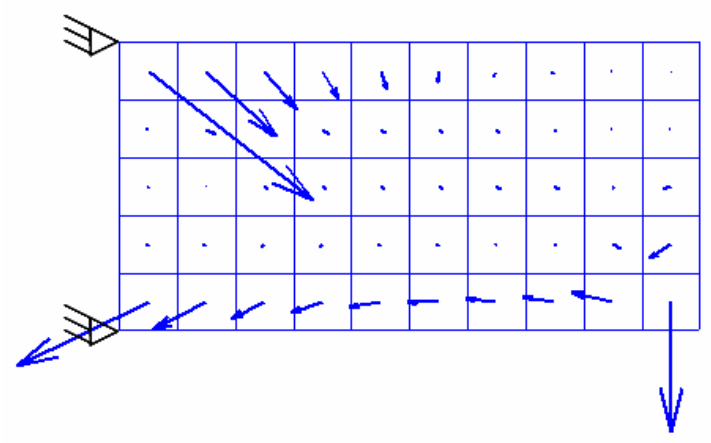

(a) Transferred force

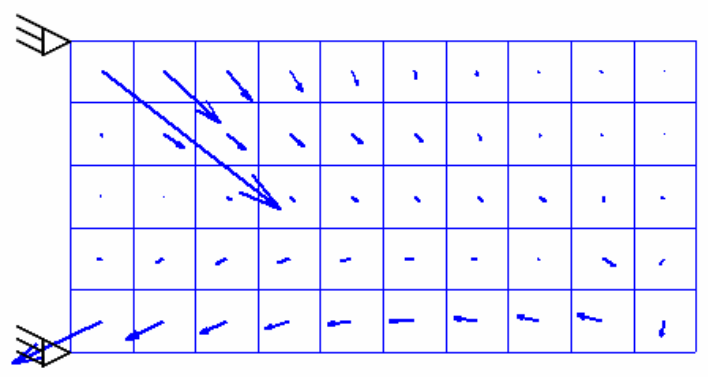

(b) Potential transferred force

Fig. 8. Transferred and potential transferred force to the reaction points with 50 elements

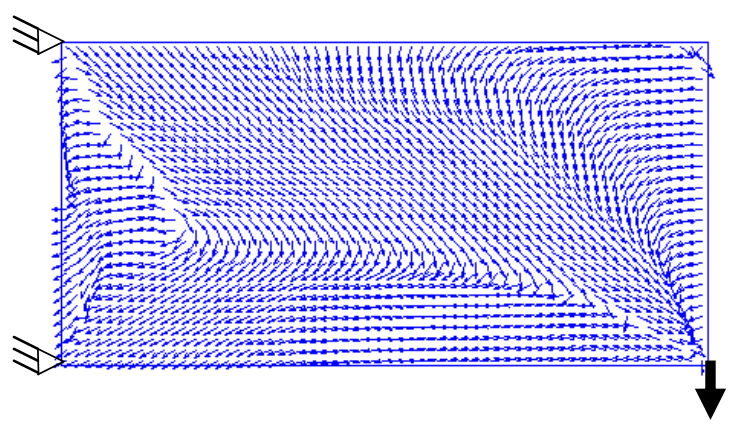

(a) Transferred force

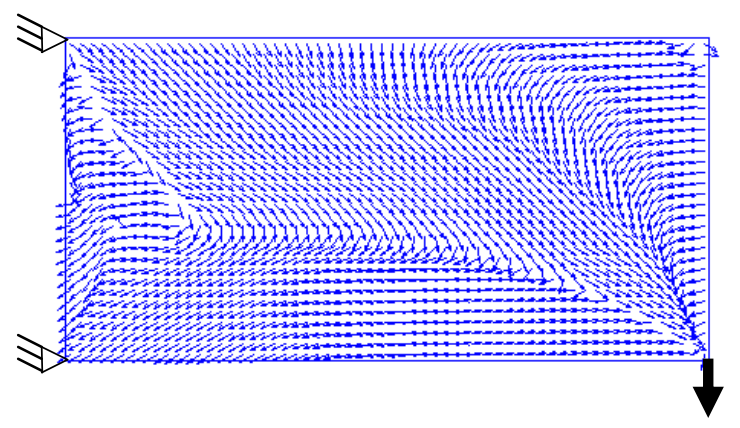

(b) Potential transferred force

Fig. 9. Orientations of the transferred and potential transferred force as unit vectors

Figure 9 shows the orientations of the transferred (a) and potential transferred force (b) as unit vectors without indicating the magnitudes. This way, the orientations of load being transferred (load paths) can be easily visualized. Both orientations are almost identical. Some differences in the orientations can be seen on the upper right corner of the structure. These differences are mainly due to numerical error because the magnitude of both transferred and potential transferred force is actually very small, approaching zero. This kind of error or difference in orientation can be noted in other cases presented in this work. The loads are visualized to be transferred from the upper and right sides of the structure toward the application point. The flow then changes direction toward the lower support. The method only provides qualitative direction 
where the loads flow. No trajectories can be traced from the point of loading application to the reaction points. By plotting the magnitude of transferred or potential transferred force, the significance of some regions in load transfer can be identified. However, the visualization does not provide critical stress regions or regions with tension and compression.

\subsection{Load path based on change in compliance of structure (U* field)}

Hoshino et al. [9] used a definition for load path based on the scalar field $U^{*}$ that provides a measure of the participation of any point in the structure to load transfer. Figure 10 shows a schematic for the procedure to calculate $U^{*}$ field. To obtain $U^{*}$, the displacements of the original structure under the applied loads are first calculated using Equation 11. The displacements calculated at the load points $u_{1 i}$ are then imposed as enforced displacement boundary conditions and the analysis is repeated. To obtain $U^{*}$ at any point $x_{i}$ in the structural domain $\Omega$, displacement degrees of freedom at point $x_{i}$ are held fixed (set to zero), and the problem is solved for displacements at other points in $\Omega$ (Equation 14).

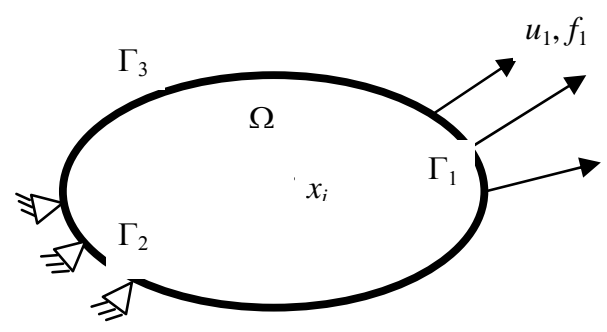

(a) Original structure with applied load $\mathrm{f}_{1}$ and calculated displacement of $u_{1}$ on $\Gamma_{1}$

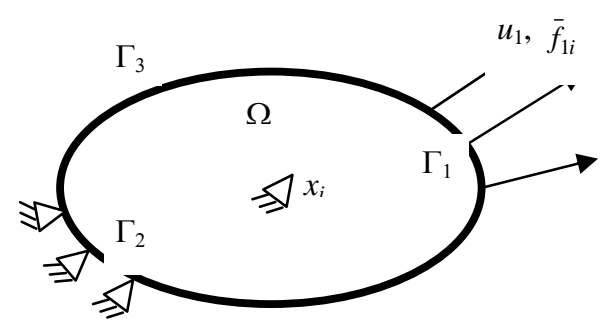

(b) Modified structure with imposed zero displacement at $x_{\mathrm{i}}$ and enforced displacement boundary condition of $u_{1}$ on $\Gamma_{1}$

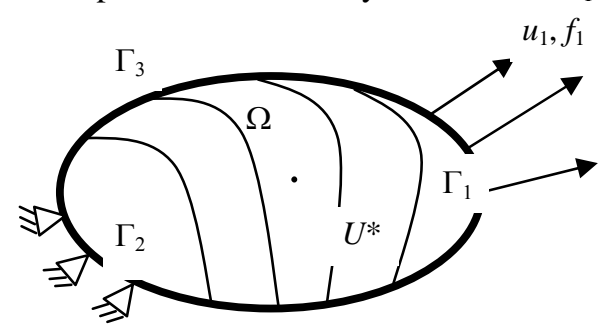

(c) Contour lines of $U^{*}$ where $U^{*}=0$ at support and $U^{*}=$ 1 at load point
Fig. 10. Schematic of procedure for calculating $U^{*}$

$$
\begin{aligned}
& \bar{\sigma}_{i j, j}+b_{i}=0 \text { for } \Omega, \\
& u_{i}=u_{1 i} \text { on } \Gamma_{1}, \\
& u_{i}=0 \text { on } \Gamma_{2}, \\
& u_{i}=0 \text { at } x_{i} \in \Omega \\
& \bar{\sigma}_{i j} n_{j}=\bar{f}_{1 i} \text { on } \Gamma_{1} .
\end{aligned}
$$

In this approach, a scalar quantity of compliance change $U^{*}$ is calculated to quantify the contribution of a point $x_{i}$ to the load transfer path. Equation 15 shows $U^{*}$ is calculated as:

$$
U^{*}=1-\frac{U}{U^{\prime}}
$$

where

$$
\begin{aligned}
& U=f_{1 i} u_{1 \mathrm{i}}, \text { and } \\
& U^{\prime}=\bar{f}_{1 i} u_{1 \mathrm{i}} \\
& f_{1 i}=\sigma_{i j} n_{j} \text { on } \Gamma_{1} . \\
& \bar{f}_{1 i}=\bar{\sigma}_{i j} n_{j} \text { on } \Gamma_{1}
\end{aligned}
$$

The gradient of this scalar field is defined as the stiffness lines. The stiffness line that provides the steepest descent in $U^{*}$ values from the load point to the support points is defined to be the load transfer path (load path) in the structure.

Hoshino et al. [9] explain that there are three conditions (termed uniformity, continuity, and consistency requirements) the $U^{*}$ field must satisfy to achieve optimum stiffness distribution in a structure. The conditions are: (i) The $U^{*}$ values along the load path must have a linear variation (uniformity); (ii) the curvature of the $U^{*}$ field with respect to the coordinate along the load path must be zero (continuity); and (iii) the load paths traced from support to loads and loads to support must be coincident (consistency). The conditions are summarized in Figure 11, where $s$ is the distance along a path from the point of application, and $l$ is the whole length of a path.

The uniformity conditions indicate that the load transferred along the load path is constant. Large curvatures of $U^{*}$ along the load path directions indicate large changes in loads transferred which implies large local shear transfer. This is the case that often arises in the presence of load path discontinuities that give rise to large stresses. Hoshino et al. [9] used the above criteria to directly tailor stiffness in automotive structures for reducing vibration. In their optimization, the area between the curve and the linear line in Figure 11 (a) is minimized along with constraints to impose the continuity and consistency requirements. They showed the optimized structure obtained corresponds to a maximum stiffness structure. 


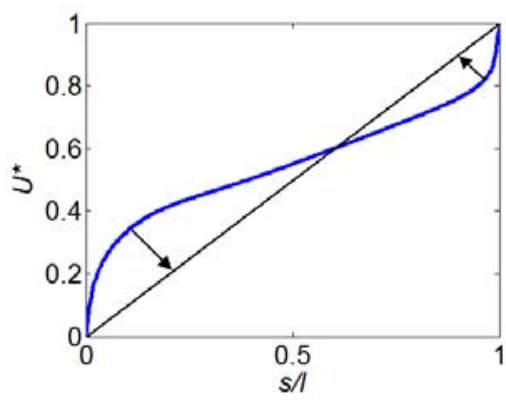

(a) Uniformity

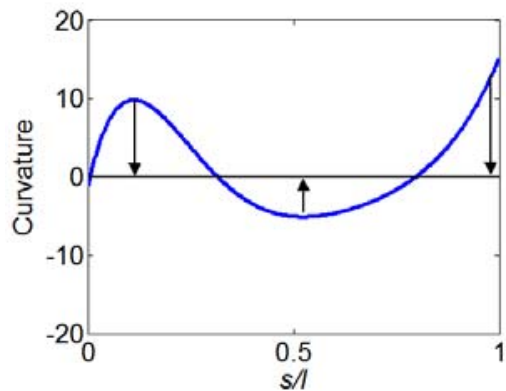

(b) Continuity

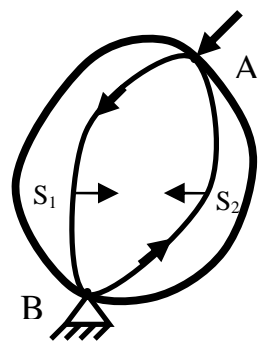

(c) Path consistency

Fig. 11. Conditions that need to be satisfied to achieve a desirable load path

Figure 12 (a) shows the contours of $U^{*}$ field for the rectangular plate with loaded end. The path corresponding to the steepest gradient of the $U^{*}$ from the support to the load is defined as the load path. The $U^{*}$ values at the boundary points are zero and one at the application point. As shown in the figure, the trajectory connects the reaction points with the point of application. Figure 12 (b) and (c) show $U^{*}$ variation along the paths and its curvature. Green indicates $U^{*}$ variation and curvature from the upper path, and blue indicates information for lower path. The figures show that the structure does not meet uniformity and continuity requirements, indicating the stiffness distribution of the plate is not optimal. Large curvatures can be seen near the supports and loading point, which indicates location of high stresses. The indication is consistent with actual location of high stresses as shown in Figure 3.

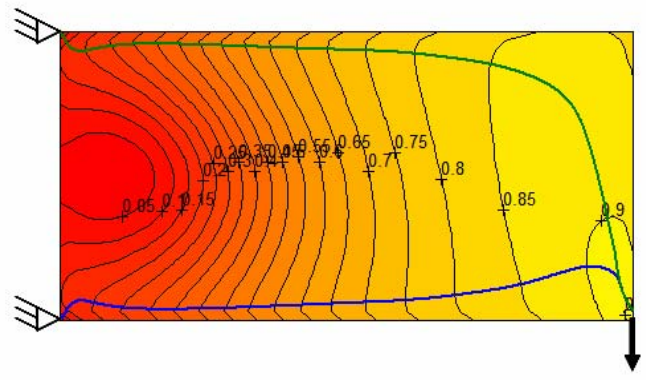

(a) Contour of $U^{*}$ and steepest gradient of $U^{*}$ as trajectory of load paths

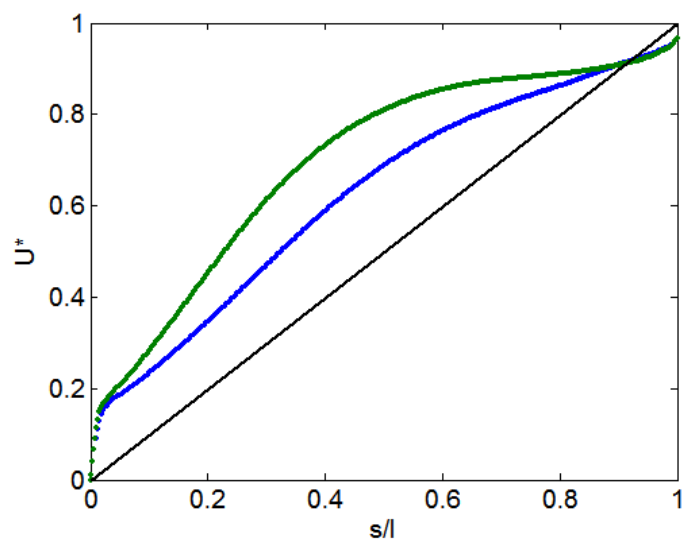

(b) $U^{*}$ variation along the paths

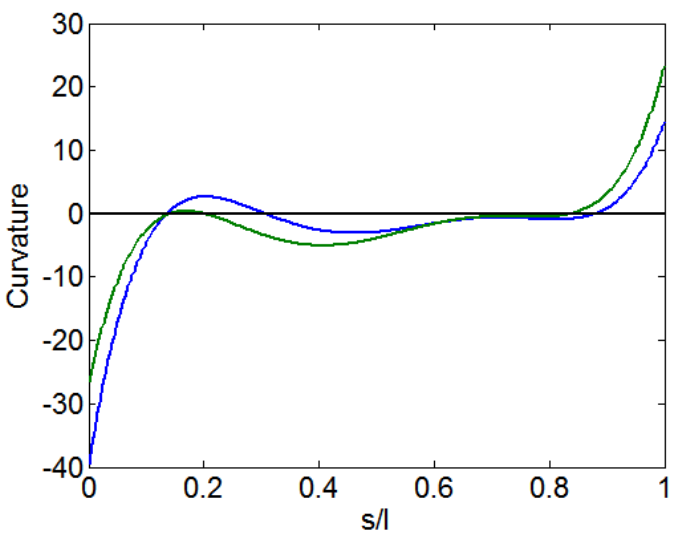

(c) Curvature of $U^{*}$ along the paths

Fig. 12. Load paths based on $U^{*}$. Green indicates upper path and blue indicates lower path.

\section{Visualizing Load Paths under Various Conditions}

It is commonly understood that alternate and different load paths arise in a structure when the structure is modified. This modification can be in the form of changes in load distributions, changes in support or boundary conditions, or changes in the form of damage that changes (reduces) locally the stiffness of the structure. This section investigates the ability of the different load path definitions presented earlier in characterizing or visualizing the 
alternate load paths that arise in the plate structure.

\subsection{Alternate load paths arising from changes in boundary condition}

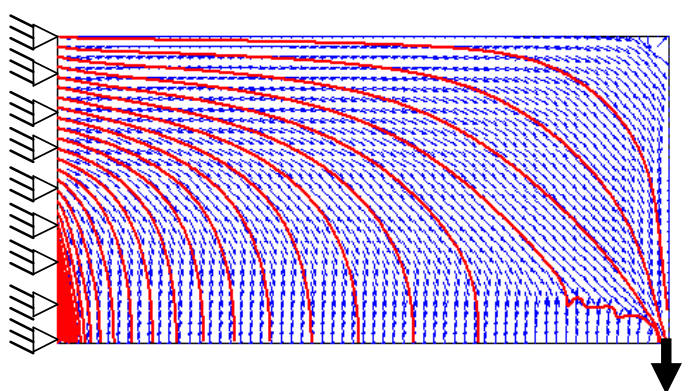

(a) Vector fields aligned with major principal stress $\sigma_{p 1}$

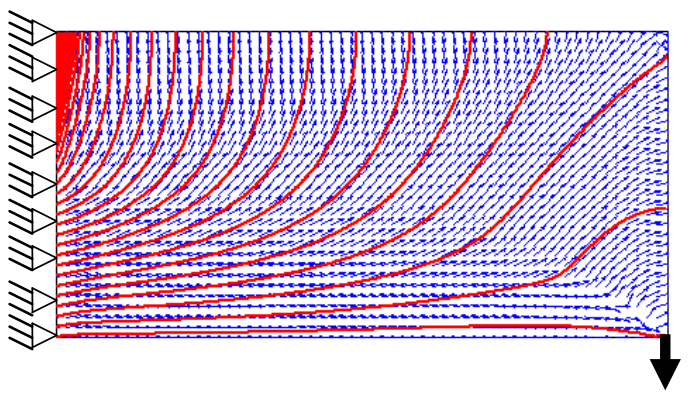

(b) Vector fields aligned with minor principal stress $\sigma_{p 2}$.

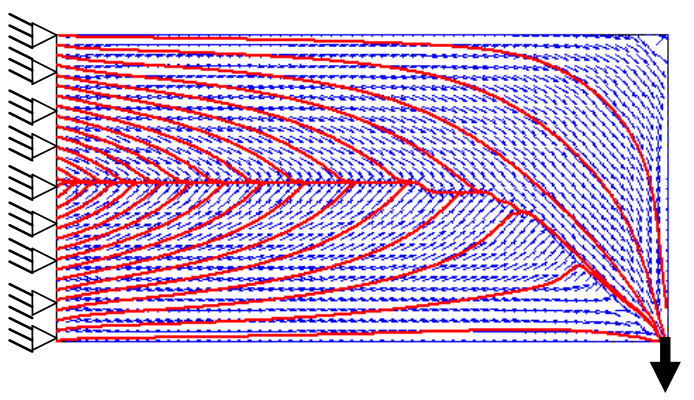

(c) Vector fields aligned with $\max \left(\operatorname{abs}\left(\sigma_{p 1}\right), \operatorname{abs}\left(\sigma_{p 2}\right)\right)$ in each element

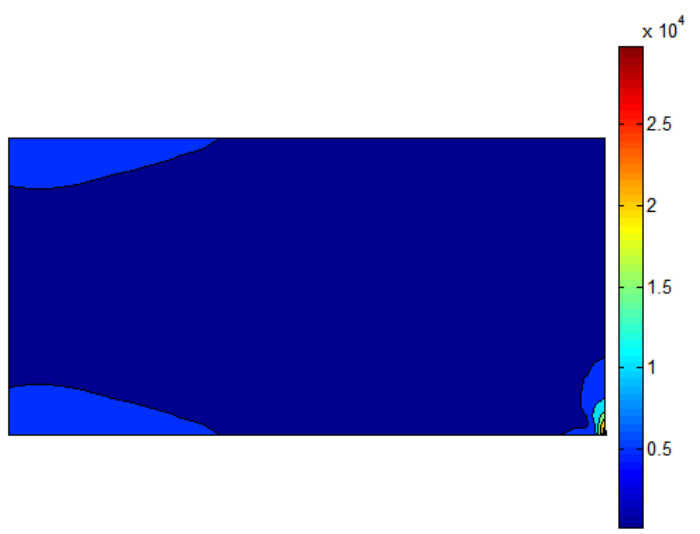

(d) Contours of magnitude of $\max \left(\operatorname{abs}\left(\sigma_{\mathrm{p} 1}\right), \mathrm{abs}\left(\sigma_{\mathrm{p} 2}\right)\right)$ in each element

Fig. 13. Load paths based on principal stresses with left side of the structure fully constrained

The support boundary condition on the left side of the structure is constrained along the entire width. In the uniform plate, the load paths will distribute the applied load to the reaction points along the fixed edge. We now investigate how the different methods differentiate the change in load paths as a result of this change in boundary condition.

Figures 13 (a) and (b) show the vector fields oriented with $\sigma_{p 1}$ and $\sigma_{p 2}$ respectively at each element in the structure. Where there are two support points, tensile stress is highest at the top of the plate near the supports, and it diminishes to zero at the bottom side of the plate (Figure 13 (a)), except near the application point. High stress areas are spread closer to the upper half of the supports. Similarly, the structure experiences high compressive stress at the bottom side, and the stress goes to zero at the top of the structure (Figure 13 (b)). High compressive stress spreads near the lower half of the supports. In general, the stresses are more spread out when the left side of the structure is fully constrained. Figure 13 (c) shows the orientations of high stresses (tension and compression) by plotting $\max \left(\left|\sigma_{\mathrm{p} 1}\right|,\left|\sigma_{\mathrm{p} 2}\right|\right)$. Figure 13 (d) shows the contours of $\max \left(\left|\sigma_{\mathrm{p} 1}\right|,\left|\sigma_{\mathrm{p} 2}\right|\right)$, which indicates high stresses near the loading point. By combining the plots of orientations of both principal stresses, we can trace trajectories that connect point of loading application and reaction points. However, these trajectories do not indicate constant load transfer or load equilibrium along the trajectories.

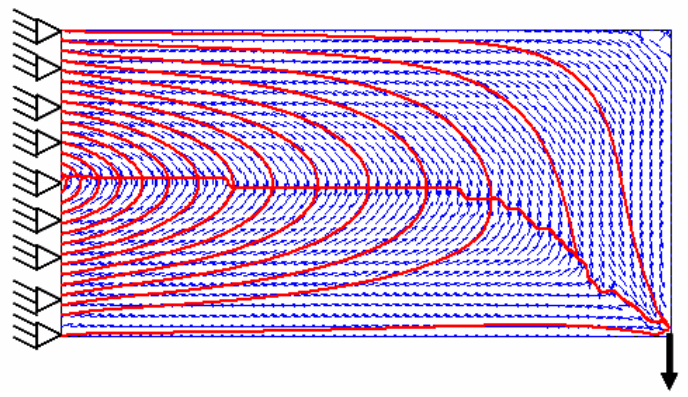

(a) Load flow with equilibrium in $x$-direction

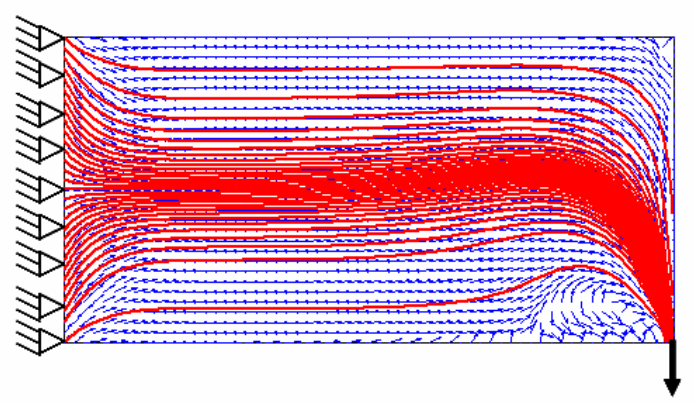

(b) Load flow with equilibrium in $y$-direction

Fig. 14. Orientation of load flow with left side of the structure fully constrained

Figure 14 shows load flow with load equilibrium in the $x$-direction (a) and the $y$-direction (b). Similar to the case of having two supports, load flow in the $x$-direction shows the structure reacts to bending moments. For load flow in the $y$ direction, shear is transferred from the application point to the reaction points. Unlike the case with only two support 
points, the region with a circulating pattern is only found near the application point. This shows that the shear is transferred more uniformly in the structure at the support. Qualitatively, the trajectories in Figure 14 (a) are very similar to the trajectories in Figure 13 (c). This indicates that dominant stress is $\sigma_{x}$, where non-zero principal stress directions are aligned with the $x$-direction.

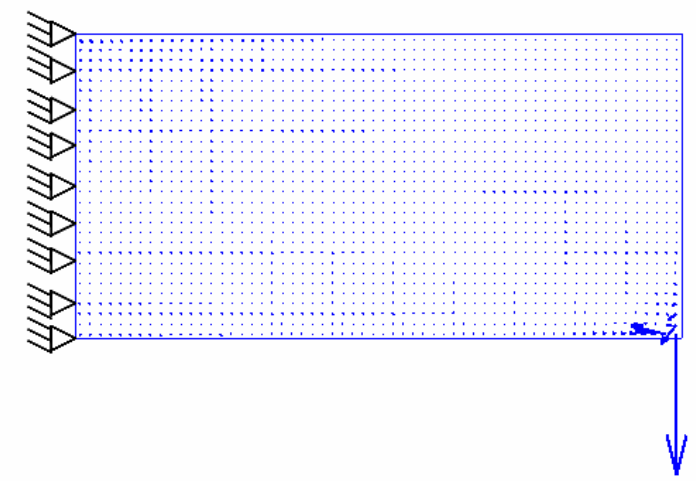

(a) Transferred force

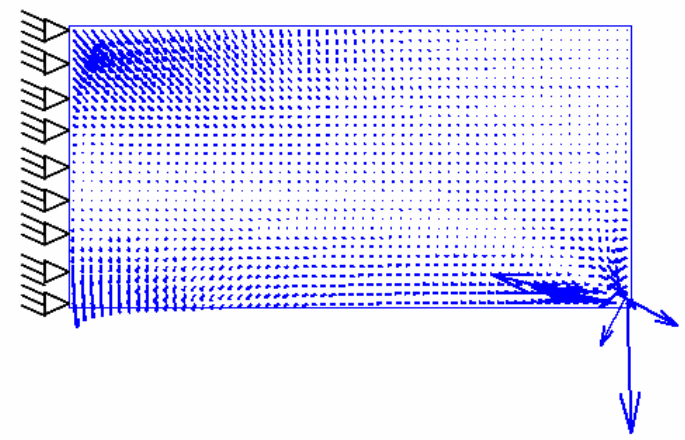

(b) Potential transferred force

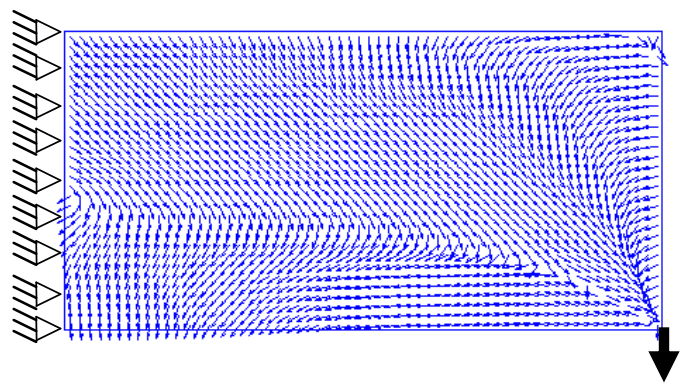

(c) Unit vectors showing orientations of transferred force

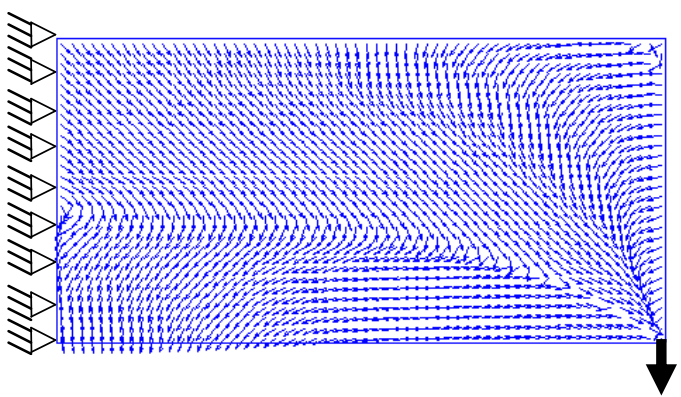

(d) Unit vectors showing orientations of potential transferred force

Fig. 15. Transferred and potential transferred force to the reaction points with left side fully constrained
Figure 15 shows transferred (a) and potential transferred (b) force when the isotropic rectangular plate is fully constrained on the left side. Plotting the transferred force shows that the only elements that contribute significantly in transferring force to the reaction points are the ones near the application point. The rest have small and almost equal contribution. This is because the force is transferred to more reaction points than in the case with only two reaction points. Plotting the potential transferred force shows many elements that can significantly change the load paths by increasing the stiffness of those elements. To visualize the flow of force being transferred, the orientations of the transferred and potential transferred force are plotted as unit vectors, shown in Figure 15 (c) and (d). Qualitatively, the orientations of transferred and potential transferred force are very similar.

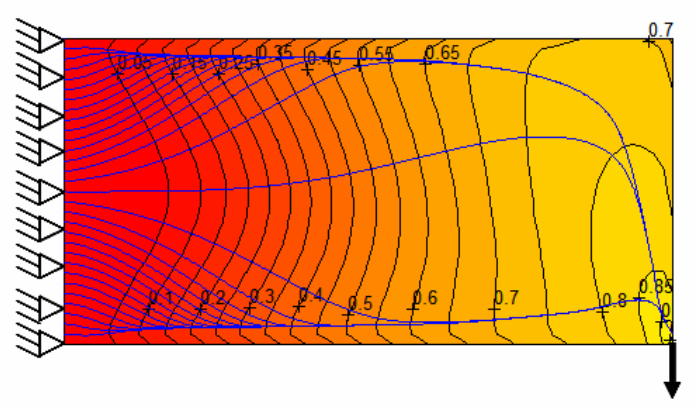

(a) $U^{*}$ field and load path trajectories

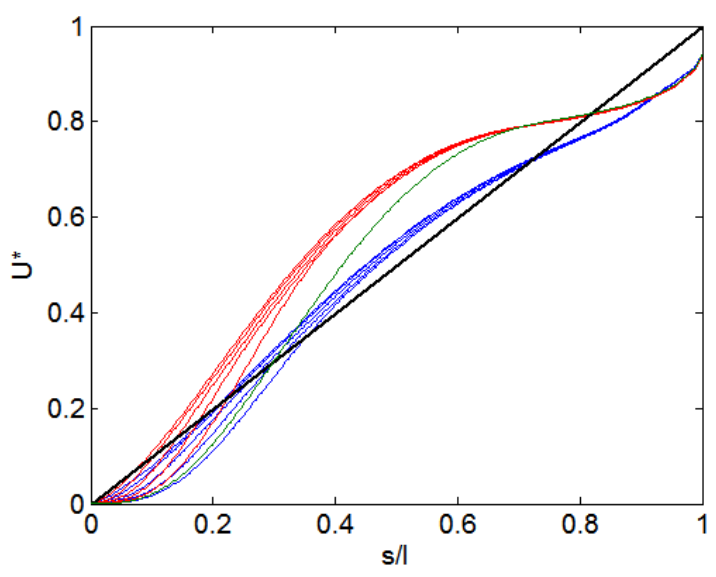

(b) $U^{*}$ variation along the load paths

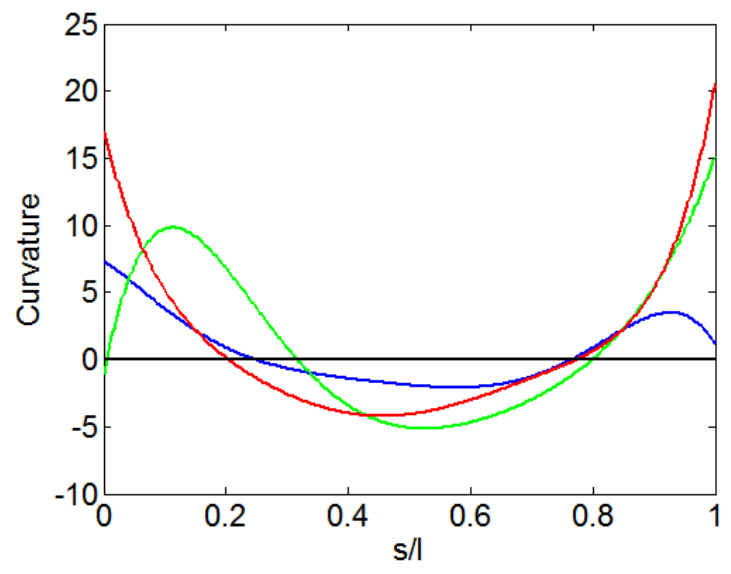

(c) Curvature of $U^{*}$ along the paths 
Fig. 16. Load paths based on $U^{*}$ for rectangular structure with its left side fully constrained. Blue indicates lower path, green indicates paths from the center support, and red indicates upper path.

Figure 16 (a) shows the $U^{*}$ field for the rectangular plate with its left side fully constrained. The load paths or steepest gradient of $U^{*}$ field are also plotted from each support to the application point. Figure 16 (b) shows the variation of $U^{*}$ along each path. The blue lines indicate load paths from the lower half of the supports, and the red lines indicate load paths from the upper half of the supports. The green line represents load path that comes from the center of the support. Load paths from the lower half have more linear variation of $U^{*}$ than load paths from the upper half. Figure 16 (c) shows the curvatures of $U^{*}$. The curvatures plotted are from paths traced from a point closed the lower support (blue), a point near the upper support (red), and a point from the center support (green). The curvatures indicate there are high stresses near the lower and upper supports. A high stress region is also indicated in the path traced from the center support at 0.11 of $s / l$. In reality high stress region is only in the region near the loading application point as shown in Figure 13 (d). Thus a relation between high stress regions/stress concentrations and discontinuity in $U^{*}$ curvature cannot be established in this case.

\subsection{Alternate load paths arising from distributed loading}

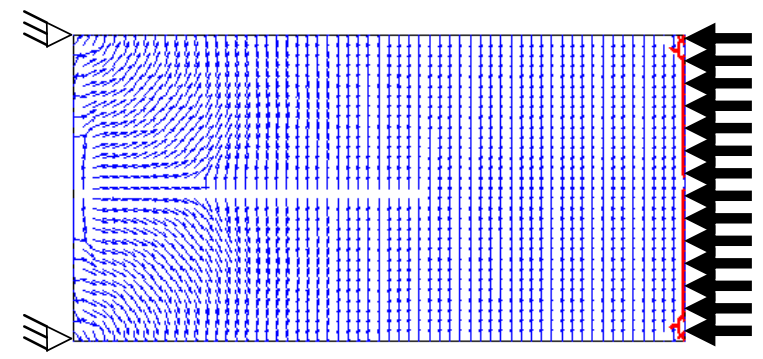

(a) Vector fields aligned with major principal stress $\sigma_{p 1}$

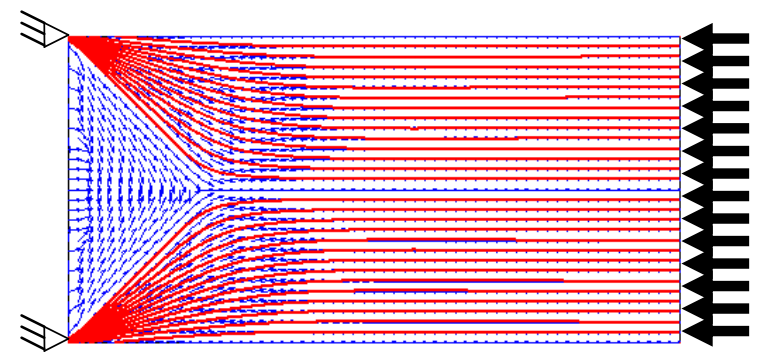

(b) Vector fields aligned with minor principal stress $\sigma_{p 2}$

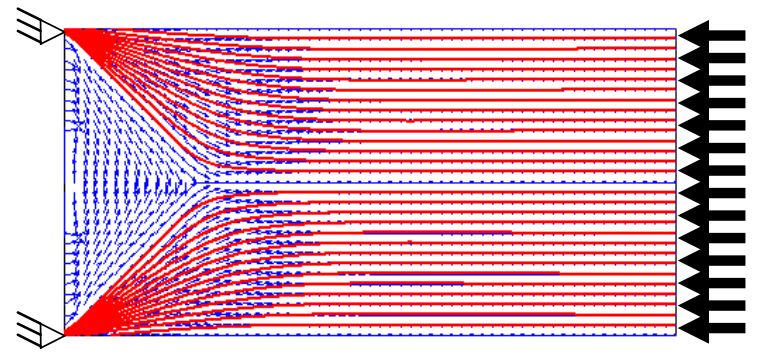

(c) Vector fields aligned with $\max \left(\operatorname{abs}\left(\sigma_{\mathrm{p} 1}\right), \mathrm{abs}\left(\sigma_{\mathrm{p} 2}\right)\right)$ in each element

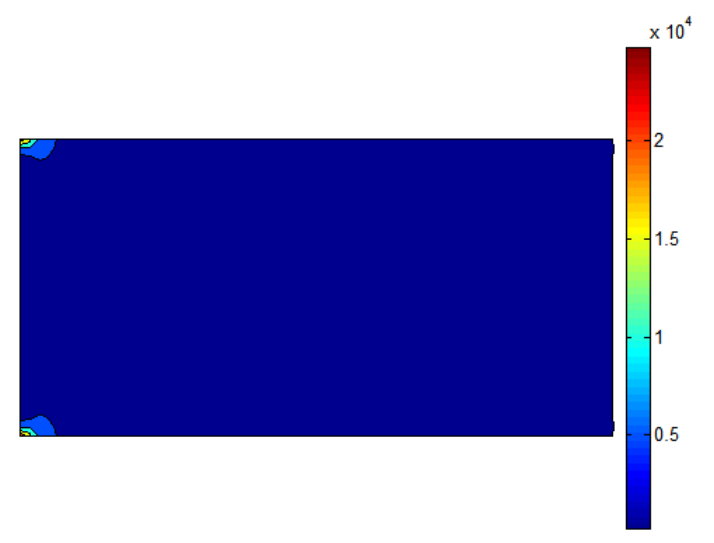

(d) Contours of magnitude of $\max \left(\operatorname{abs}\left(\sigma_{p 1}\right), \operatorname{abs}\left(\sigma_{p 2}\right)\right)$ in each element

Fig. 17. Load paths based on principal stresses with distributed loading in the horizontal direction

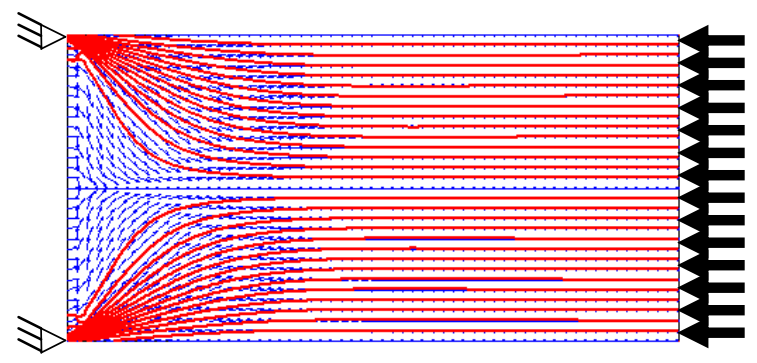

(a) Load flow with equilibrium in $x$-direction

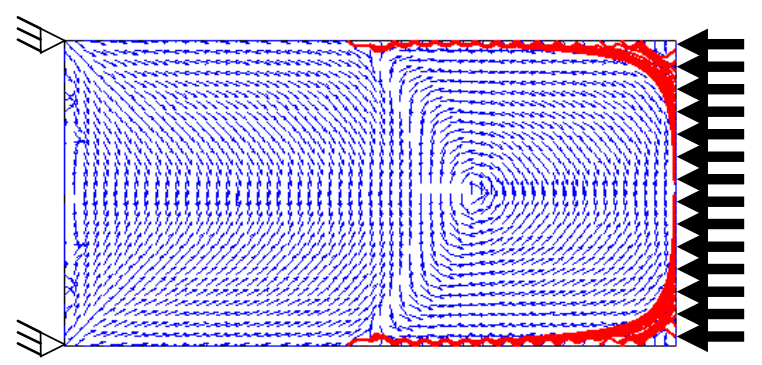

(b) Load flow with equilibrium in $y$-direction

Fig.18. Orientation of load flow with distributed loading in the $x$-direction 
The load path definitions are also compared to visualize load paths caused by distributed loading in the structure. Figure 17 shows the orientations of principal stresses. In the regions where orientations of minor principal stresses are parallel to the loading direction, the major principal stress is zero. Since the distributed loads create compression in the plate, initially vectors of the minor principal stress (compression) are parallel to the loading direction then turn toward the supports (Figure 17 (b)). Trajectories can also trace the stress flow from the loading points to the supports. The highest compression occurs near the supports. Figure 17 (c) shows the orientation of $\max \left(\operatorname{abs}\left(\sigma_{\mathrm{p} 1}\right), \mathrm{abs}\left(\sigma_{\mathrm{p} 2}\right)\right)$ in each element, which is almost identical to Figure 17 (b). This shows that the dominant stress in the structure is compressive stress. Figure 17 (d) shows the contours of $\max \left(\operatorname{abs}\left(\sigma_{\mathrm{p} 1}\right), \operatorname{abs}\left(\sigma_{\mathrm{p} 2}\right)\right)$ in each element, which shows regions with high stresses are near the supports.

Figure 18 (a) shows that the load flow in the $x$-direction corresponds to the orientations of the minor principal stress. The same trajectories trace load flow from the loading points to the reaction points. Load flow in the $y$-direction plotted in Figure 18 (b), which complements load flow in the $x$-direction, shows circulating patterns in the right half of the plate. These patterns occur because of the equilibrium requirement explained by Kelly et al. [3]. Waldman et al. [4] explained that these circulatory patterns are self equilibrating because the $y$-components of the loads are zero. Orientations of minor principal stresses and orientations of load flow with equilibrium in $x$-direction are very similar (including their trajectories). Under distributed loads, the structure experiences compression along the $x$ direction. That is why orientations from minor principal stress (which indicate compression in the structure) are very similar to orientations from load flow in $x$-direction, which is again aligned with the loading direction.

With distributed loading in the $x$-direction, plotting the transferred and potential transferred force distributions indicates regions where load transfer is most significant and the potential effectiveness of strengthening some regions to improve load transfer. In general, regions next to the supports exhibit the highest amount of load transfer (Figure 19 (a)). Strengthening these regions consistently improves stiffness and load transfer (Figure 19 (b)). Thus both reducing and increasing stiffness of elements near the supports are effective in changing the load transfer (load paths). Figure 19 (c) and (d) show the orientations of transferred and potential transferred force more clearly. Both figures show that the orientations are almost identical.

Figure 20 (a) shows the contours of $U^{*}$ field and the trajectory where the steepest gradients of the contours occur (load paths). Tracing trajectories from lower and upper supports shows that both paths meet at one point at the right side of the structure, where the distributed loads are applied. An explanation for this is that $U^{*}$ at the supports is zero and $U^{*}$ along the side where the loads are applied is one. Near the side where loads are applied there is only one location at each contour where the gradient of $U^{*}$ is steepest. Thus, both paths meet at one location in the middle of the application side. Analyzing the $U^{*}$ variation along both paths shows the variation to be identical in both paths (Figure 20 (b)). Figure 20 (c) shows the curvature of $U^{*}$ along the paths, which is mostly zero with exception near the supports and loading points. Ideally, the curvature near the applied loads should be zero because there are no high stress regions near where the loads are applied. This is the drawback of using $U^{*}$ method for distributed loading. A complimentary concept of $U^{*}$ (a new index $U^{* *}$ ) is introduced by Okano et al. [10] that can be used to investigate load paths in structures under distributed loading. The new index $U^{* *}$ is defined in terms of the complimentary strain energy instead of the work done by the load (compliance) in case of $U^{*}$.

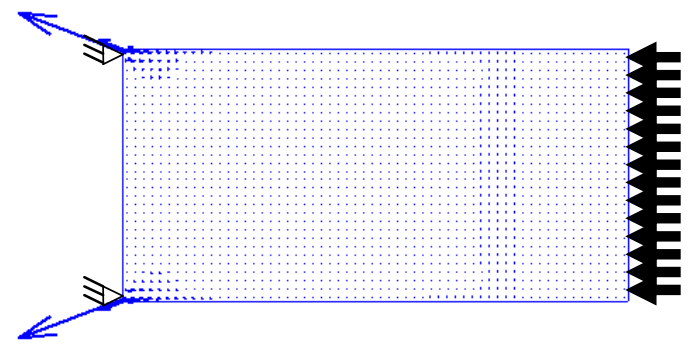

(a) Transferred force

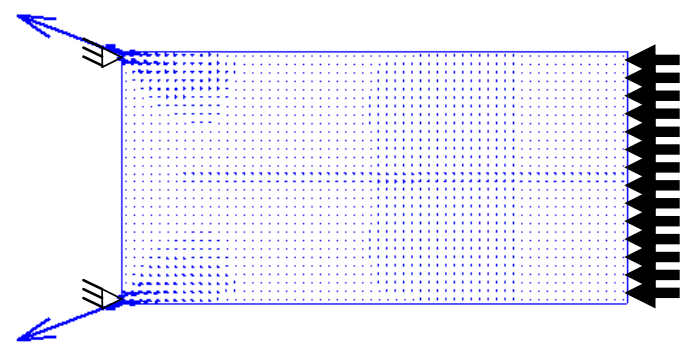

(b) Potential transferred force

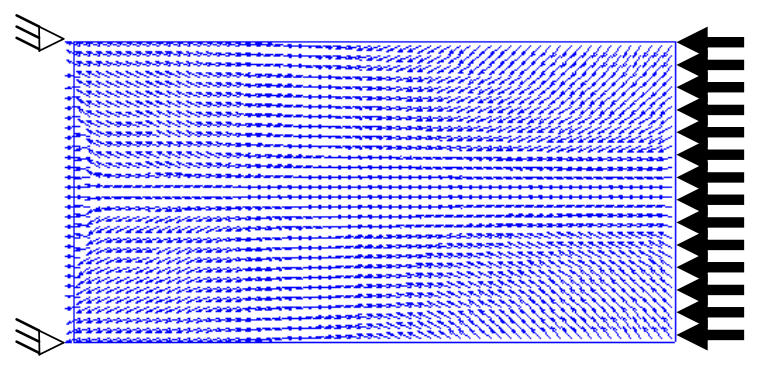

(c) Unit vectors showing orientations of transferred force

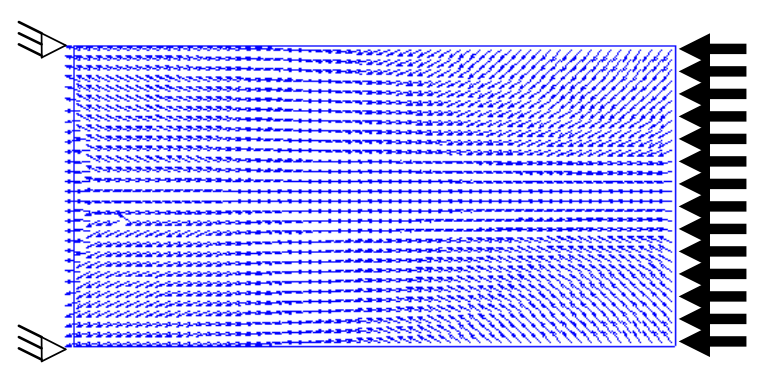

(d) Unit vectors showing orientations of potential transferred force

Fig. 19. Transferred and potential transferred force to the reaction points with distributed loading in the $x$-direction 


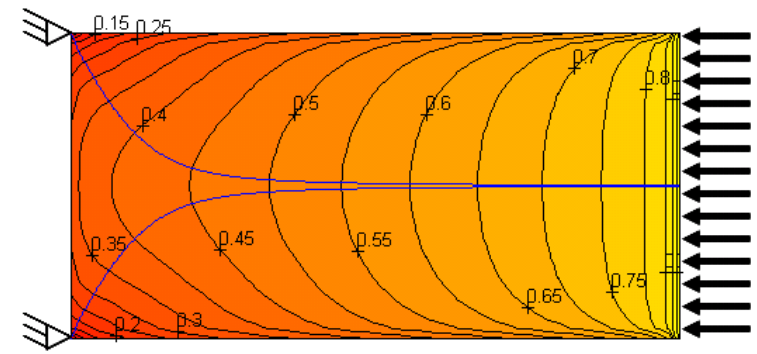

(a) $U^{*}$ field and load path trajectories

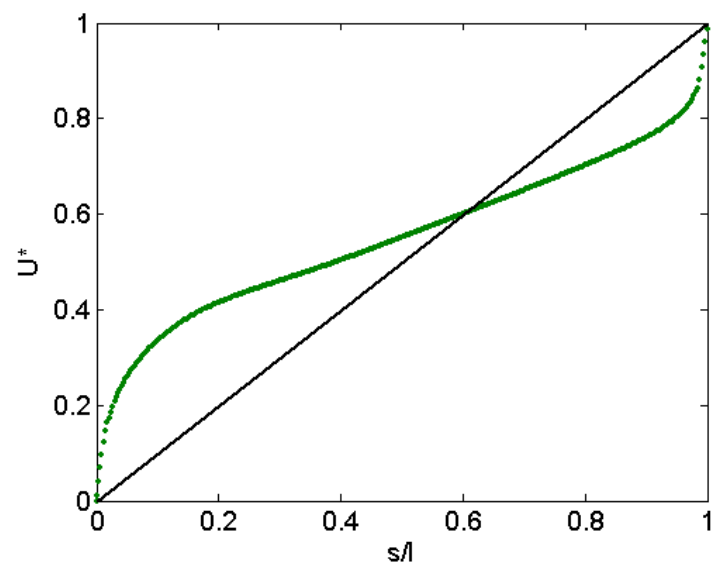

(b) $U^{*}$ variation along the load paths

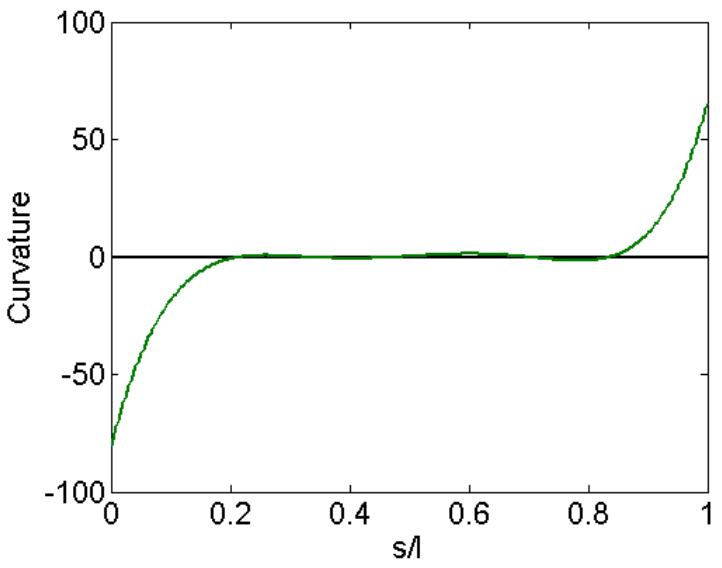

(c) Curvature of $U^{*}$ along the paths

Fig. 20. Load paths based on $U^{*}$ for rectangular structure with distributed loading in the $x$-direction

\subsection{Alternate load paths arising from introducing a hole}

Engineers are particularly interested to see how load paths change in the events of damage to the structure. The idea is to check whether the alternative paths will enable the structure to perform its basic function. In this work, the damage introduced to the structure is a circular hole. Comparisons are made on the different ways the four load path definitions visualize alternative load paths.

Figure 21 shows the orientations of the principal stresses. Figure 21 (a) shows the orientation of the major principal stress vectors (tensile). As in the non-damage case, the plot of major principal stress orientations shows tension on the top side of the plate and the stress diminish to zero at the bottom side. Maximum tension occurs at the upper left support. Figure 21 (b) shows that the structure experiences compression at the bottom side (maximum compressive stress at the lower left support) and the compressive stress decreases to zero at the top side. The findings about orientation of $\sigma_{p 1}$ and $\sigma_{p 2}$ around the hole are similar to the results obtained by Waldman et al. in [4]. They noted that depending on the location along the free boundary of the hole, one of the two principal stresses will be zero. The nonzero principal stresses will be oriented tangent to the boundary of the hole. Due to the inherent numerical error in finite element method, the areas where orientations of the principal stresses are shown to be perpendicular to the hole are in fact areas with zero principal stresses. The trajectory from the upper support can be seen closely follows the boundary of the hole where the orientations of $\sigma_{p 1}$ are tangent to the hole boundary, and ends at the application point. On the other hand, trajectory from the lower support ends at the boundary of the hole where the orientations of the $\sigma_{p 2}$ are perpendicular to the hole boundary (where $\sigma_{p 2}$ is essentially zero). This is a major deficiency in using orientations of principal stresses because it is impossible for the load trajectory to end at the boundary of the hole or for the hole to transmit loads.

Plotting the orientation of $\max \left(\operatorname{abs}\left(\sigma_{\mathrm{p} 1}\right), \mathrm{abs}\left(\sigma_{\mathrm{p} 2}\right)\right)$ in each element (Figure 21 (c)) shows how the stresses go around the hole. The orientations of $\sigma_{p 1}$ and $\sigma_{p 2}$ that are now plotted are the ones tangent to the surface of the hole. Tracing trajectory from the lower support ends at the loaded end, and tracing another one from the upper support ends at the right side of the plate. Figure 21 (d) shows the contour of magnitude of maximum principal stresses. High stresses are indicated to be near the supports and loading point.

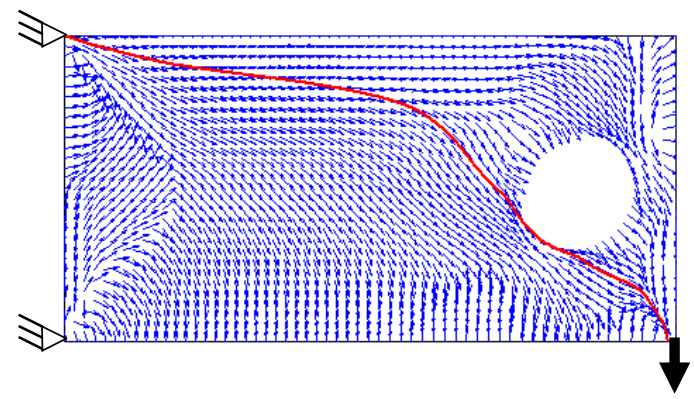

(a) Vector fields aligned with major principal stress $\sigma_{p 1}$

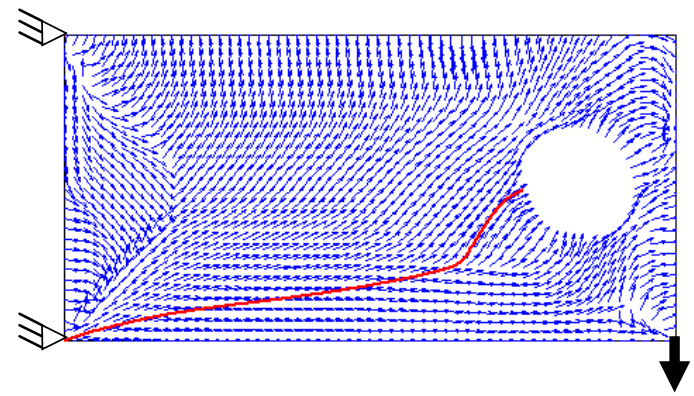

(b) Vector fields aligned with minor principal stress $\sigma_{p 2}$ 


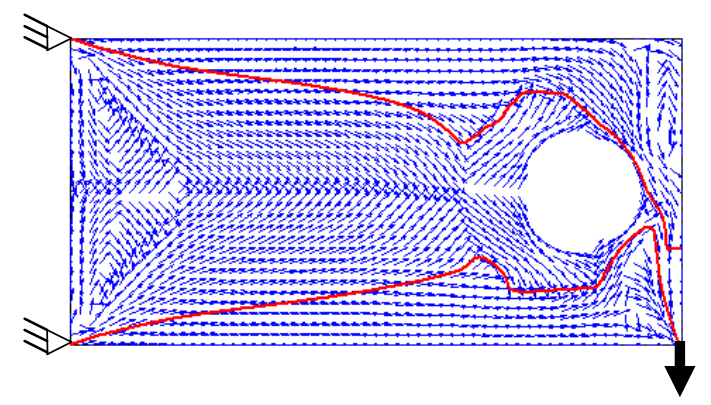

(c) Vector fields aligned with $\max \left(\operatorname{abs}\left(\sigma_{\mathrm{p} 1}\right), \mathrm{abs}\left(\sigma_{\mathrm{p} 2}\right)\right)$ in each element

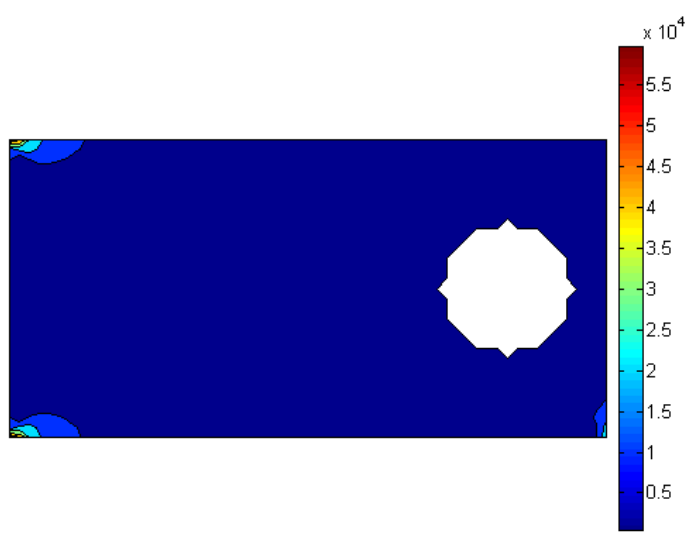

(d) Contours of magnitude of $\max \left(\operatorname{abs}\left(\sigma_{p 1}\right), \operatorname{abs}\left(\sigma_{p 2}\right)\right)$ in each element

Fig. 21. Load paths based on principal stresses with a hole in the structure

Figure 22 (a) shows the distribution of load flow with load equilibrium in the $x$-direction. Similar to the case with no hole in the structure, the flow indicates bending in the structure. The hole changes the loops (red line trajectories) that used to connect between the two supports and to the application point. The load now flows around the hole, and end at the right side of the structure. There is also a region with circulatory patterns on the right side of the structure, which indicates region with low stress. Figure 22 (b) shows the distribution of load flow with load equilibrium in the $y$ direction. Similar to the case with no damage, shear is still transferred from the point load to the reaction points. The load has to travel around the hole. The hole creates more regions with circulatory patterns (near the hole) which have to satisfy the equilibrium requirements.

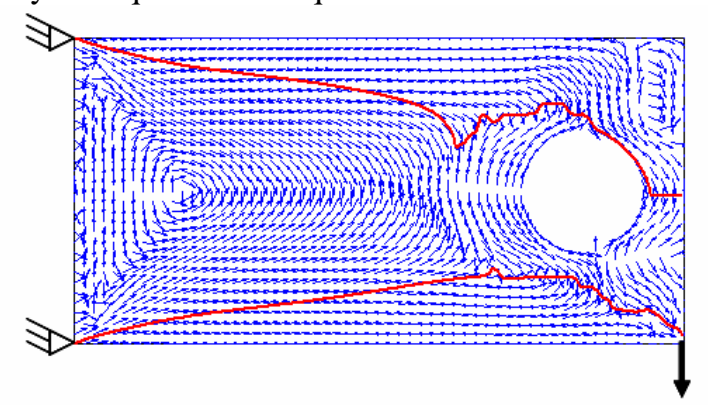

(a) Load flow with equilibrium in $x$-direction

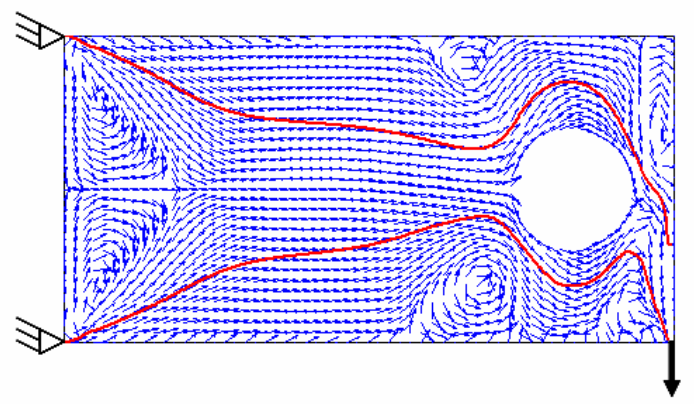

(b) Load flow with equilibrium in $y$-direction

Fig. 22. Orientation of load flow with a hole

The case with a hole for transferred and potential transferred force is shown in Figure 23. A quick inspection shows that differences in transferred and potential transferred force distribution between the case with a hole and no hole are subtle. An explanation for this is that the hole exists in the area where the elements contribute little to transferring force to the reaction points. Thus creating the hole does not make significant changes in the transferred and potential transferred force in other elements. This example gives the basis for removing materials in the structure during redesign process in later work by Harasaki and Arora [7 and 8]. By plotting the orientations of transferred and potential transferred force as unit vectors (Figure 23 (c) and (d)), we can visualize how the flow of transferred loads avoid the hole. Qualitatively, the orientations of transferred and potential transferred load are similar.

Using $U^{*}$ field to identify load paths from the application point to the reaction points with a hole in the structure shows that the paths simply avoid the hole by taking a longer route (Figure 24 (a)). Changes in the paths are also evident in the plot of $U^{*}$ variation along the paths (Figure 24 (b)). Non-linear variation of $U^{*}$ along the paths indicates turns in the paths. The curvatures of $U^{*}$ indicate high stresses near the supports and loading point by having large values of curvature. This observation is consistent with the actual location of high stress regions.

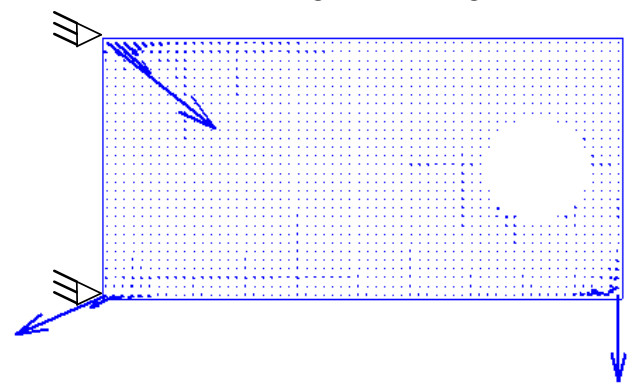

(a) Transferred force 


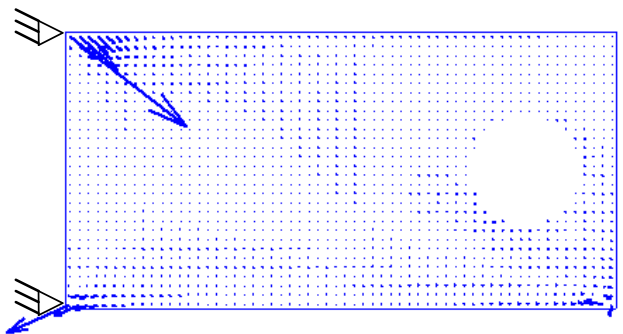

(b) Potential transferred force

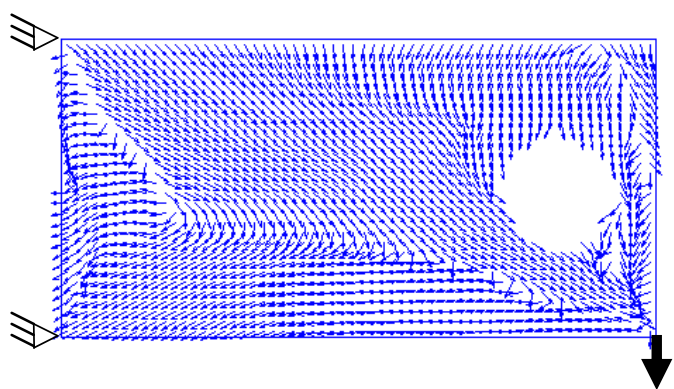

(c) Unit vectors showing orientations of transferred force

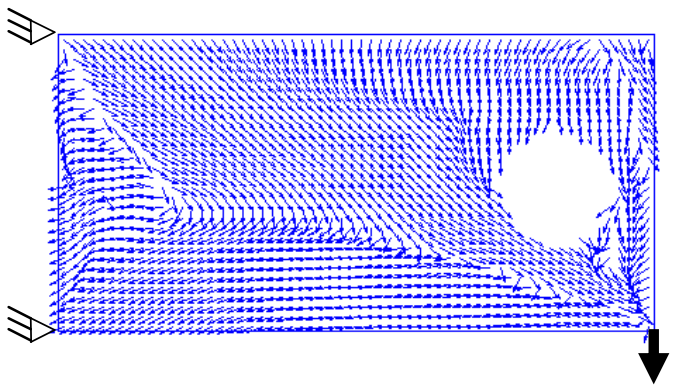

(d) Unit vectors showing orientations of potential transferred force

Fig. 23. Transferred and potential transferred force to the reaction points with a hole in the structure

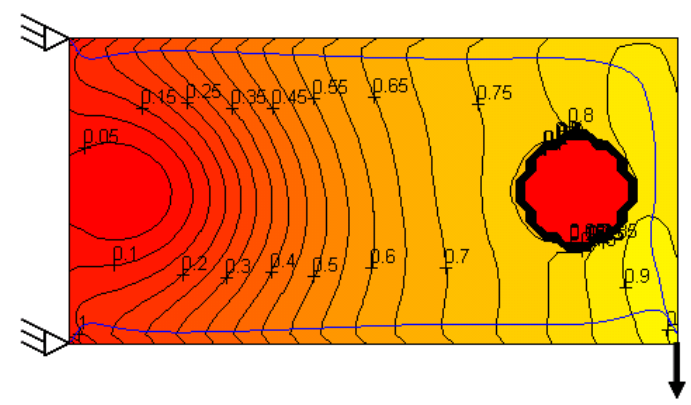

(a) $U^{*}$ field and load path trajectories

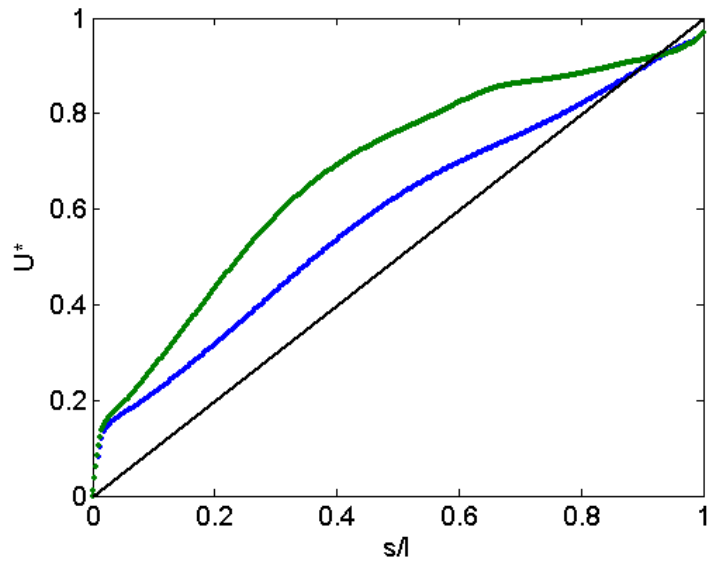

(b) $U^{*}$ variation along the load paths

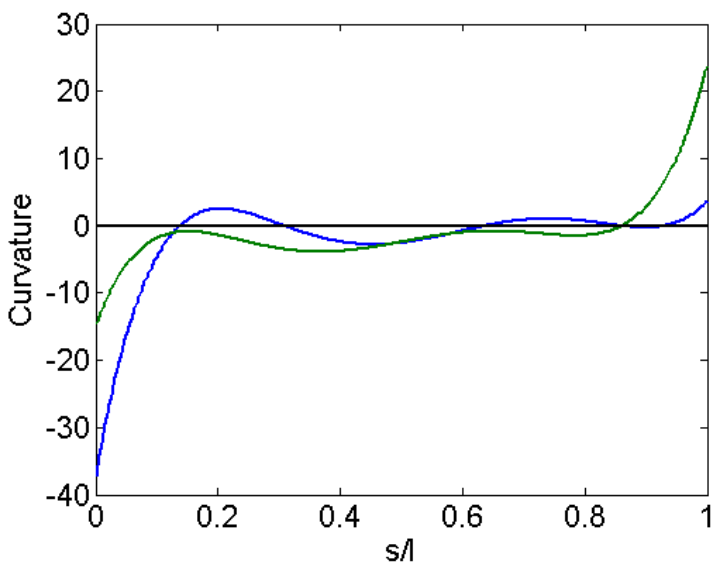

(c) Curvature of $U^{*}$ along the paths

Fig. 24. Load paths based on $U^{*}$ for rectangular structure with a hole in the structure

\subsection{Alternate load paths arising from introducing a hole and having distributed loading}

The load path definitions are compared to visualize alternate paths in the structure under distributed loading and when there is a hole. Figure 25 shows the orientation of the principal stresses under this condition. As in the case with distributed loading, the structure experiences compressive stress. On the upper and lower parts of the hole, $\sigma_{p 1}$ is mostly zero, and the orientations are also perpendicular to the hole boundary. In other parts of the structure, $\sigma_{p 1}$ is mostly zero, where the orientations are perpendicular to the direction of applied loads (Figure 25 (a)). Vectors of the minor principal stress (compressive) can be seen parallel to the loading direction initially. They pass the hole, and turn toward the supports. This is evident by tracing the trajectories from the application side to the supports in $\begin{array}{lll}\text { Figure } 25 & \text { (b). Plotting orientations of }\end{array}$ $\max \left(\operatorname{abs}\left(\sigma_{\mathrm{p} 1}\right), \operatorname{abs}\left(\sigma_{\mathrm{p} 2}\right)\right)$ in Figure 25 (c) shows that the trajectories are identical to the ones in Figure 25 (b). Clearly, the dominant stress in the structure is compressive stress. Also, the orientations of $\sigma_{p 1}$ and $\sigma_{p 2}$ can now be seen all tangent to the surface of the hole. Figure 25 (d) shows that high stresses are only near the supports. 


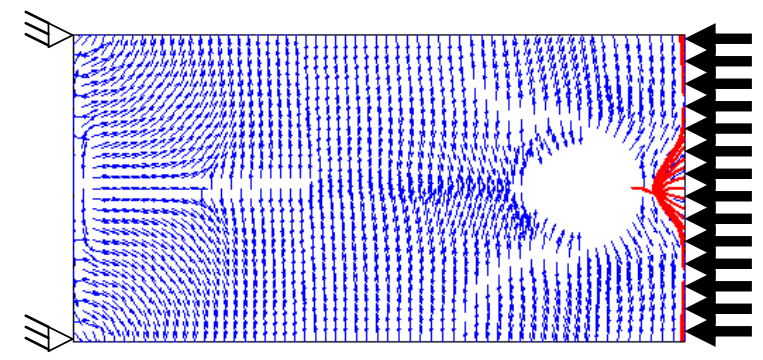

(a) Vector fields aligned with major principal stress $\sigma_{p 1}$

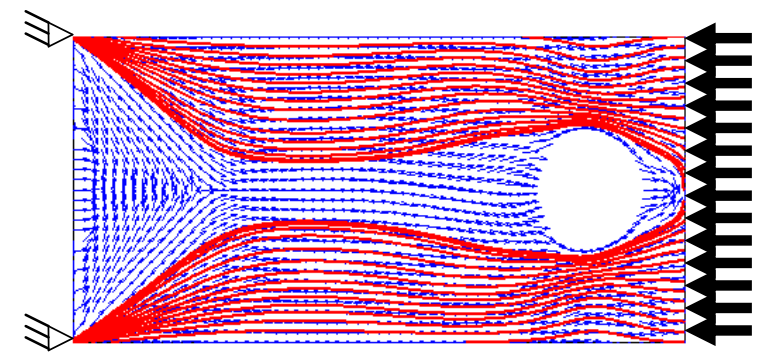

(b) Vector fields aligned with minor principal stress $\sigma_{p 2}$

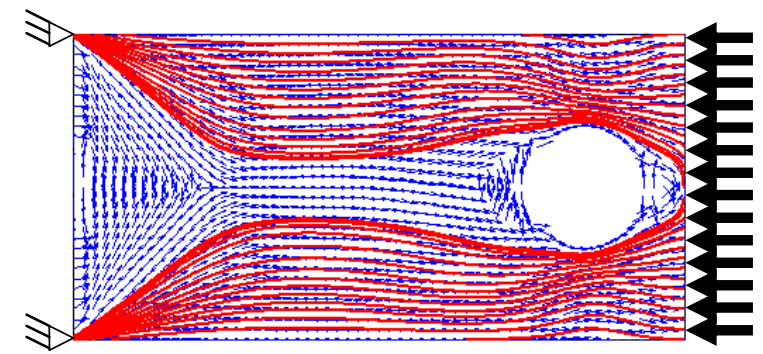

(c) Vector fields aligned with $\max \left(\operatorname{abs}\left(\sigma_{\mathrm{p} 1}\right), \mathrm{abs}\left(\sigma_{\mathrm{p} 2}\right)\right)$ in each element

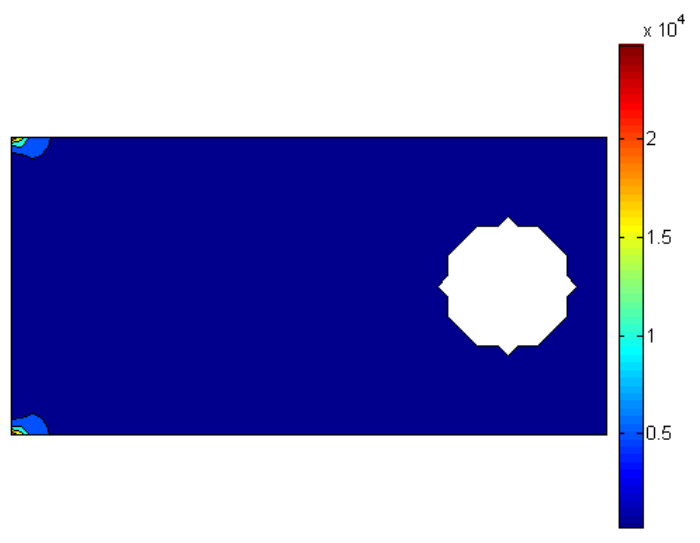

(d) Contours of magnitude of $\max \left(\operatorname{abs}\left(\sigma_{p 1}\right), \operatorname{abs}\left(\sigma_{p 2}\right)\right)$ in each element

Fig. 25. Load paths based on principal stresses with a hole and distributed loading in the structure

Again, the load flow with equilibrium in $x$-direction is the counterpart of minor principal stress (Figure 26 (a)). The patterns are more complex for load flow with equilibrium in $y$-direction (Figure 26 (b)). There are many regions with circulatory patterns in the $y$-direction load flow, especially near the hole. The circulatory patterns are self equilibrating because the $y$-components of the loads are zero.

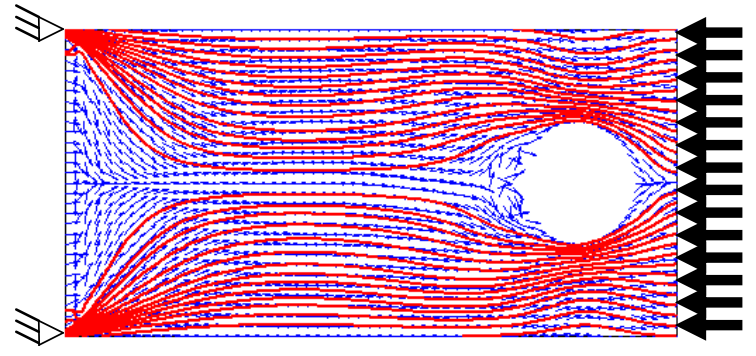

(a) Load flow with equilibrium in $x$-direction

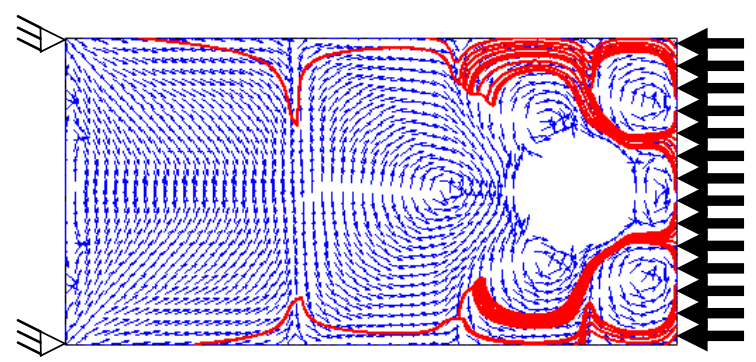

(b) Load flow with equilibrium in $y$-direction

Fig. 26. Orientation of load flow with a hole and distributed loading

Similar to the case with no hole, distributions of transferred and potential transferred force show that elements next to the supports transfer the most load (Figure 27 (a)). Strengthening these regions consistently improves stiffness and load transfer (Figure 27 (b)). The differences between the case with hole and no hole are subtle. This is because the hole is in the region where the contributions of elements in transferring loads are minimal. Thus removing elements to create the hole does not significantly change overall load transferred by the other elements. Figure 27 (c) and (d) present orientations of transferred and potential transferred force as unit vectors. Both figures visualize the flow of force being transferred across the structure and around the hole. Based on the orientations, no trajectories can be traced to connect loading point and reaction points. Also, the information obtained does not indicate regions with high stresses. Using the magnitude of the transferred and potential transferred force enables stiffness tailoring of the structure by removing material from regions with low transferred and potential transferred force.

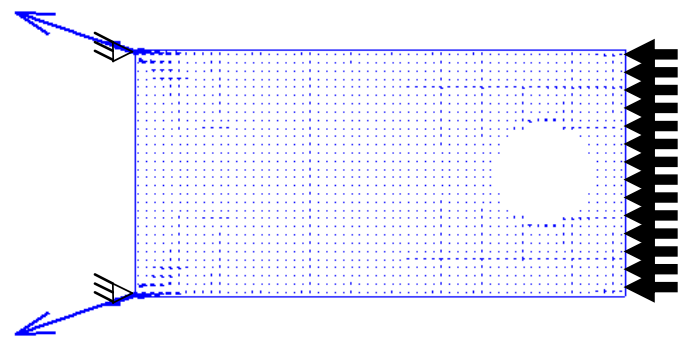

(a) Transferred force 


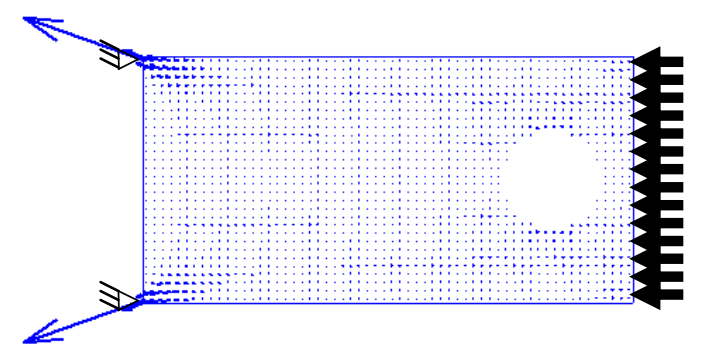

(b) Potential transferred force

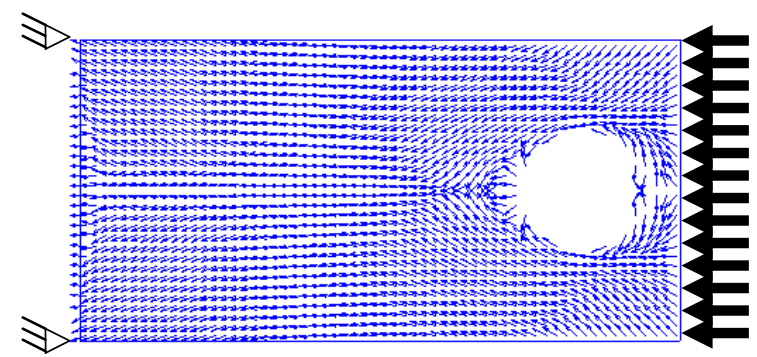

(c) Unit vectors showing orientations of transferred force

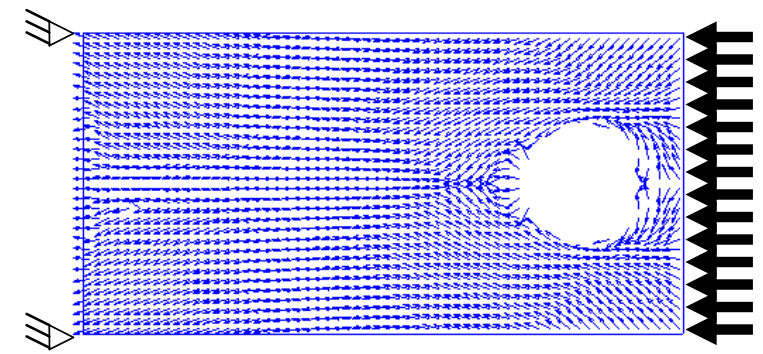

(d) Unit vectors showing orientations of potential transferred force

Fig. 27. Transferred and potential transferred force to the reaction points with a hole and distributed loading in the structure

Figure 28 (a) shows the contours of $U^{*}$ field for the plate structure with a hole and distributed load. The load paths trace trajectories from the supports, around the hole, and to the application side. Since the structure is symmetric, the $U^{*}$ variation along both lower and upper paths are identical as shown in Figure 28 (b). The two paths also end at two locations at the loading application side, where $U^{*}$ value is one along the side. The slope of $U^{*}$ variation changes at the location where the hole exists (Figure 28 (b)), which also indicates the paths turn to go around the hole. Another drawback of using $U^{*}$ method to analyze load paths in a structure under distributed loading is shown here. High stresses are only observed near the supports. However, large non-zero values of curvature near the supports and loading points indicate otherwise.

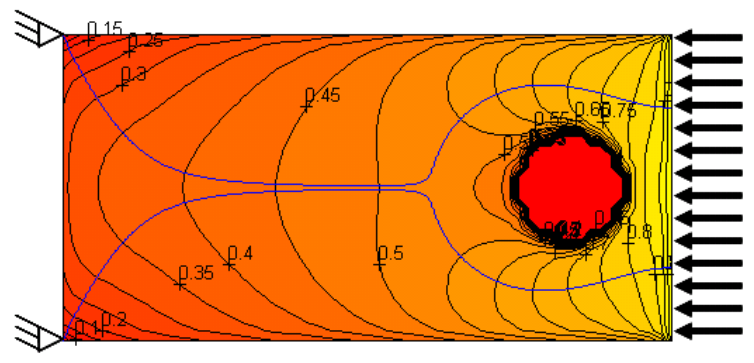

(a) $U^{*}$ field and load path trajectories

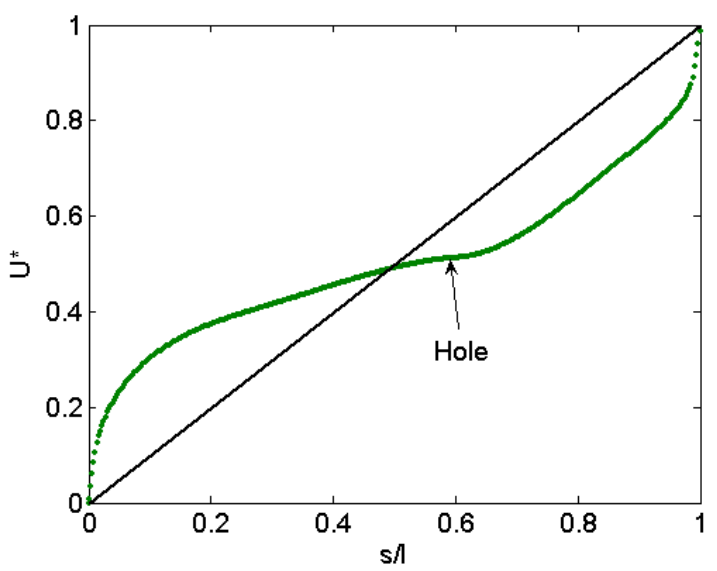

(b) $U^{*}$ variation along the load paths

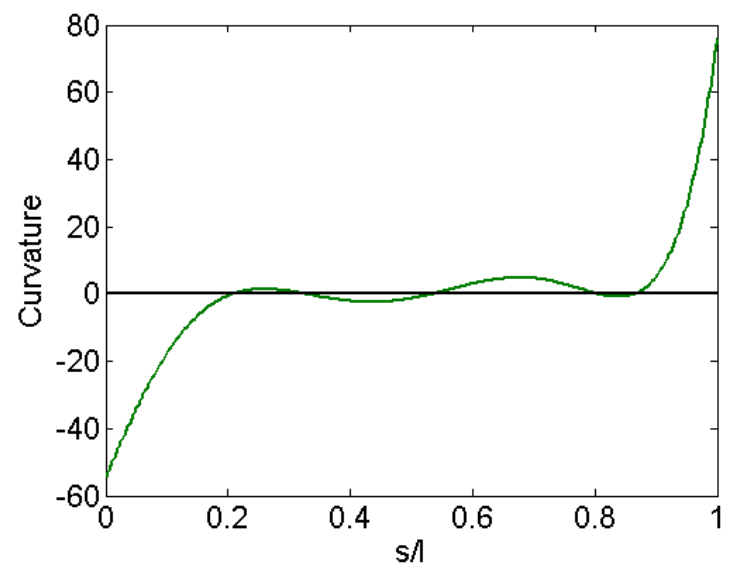

(c) Curvature of $U^{*}$ along the paths

Fig. 28. Load paths based on $U^{*}$ for rectangular structure with a hole and distributed loading in the structure

\section{Summary and Conclusions}

In summary the different definitions and methods that have been developed to characterize and visualized load paths in a structure were compared. The methods compared are: (1) using directions of major and minor principal stresses, (2) load flow defined by orthogonal axes in which load equilibrium must be satisfied, (3) transferred and potential transferred force method, and (4) load path based on change in compliance ( $U^{*}$ field). The different methods are compared for characterizing load paths in a rectangular 
plate structure under various conditions, namely: (a) two points and fully constrained boundary conditions, (b) distributed loading in $x$-direction, (c) a hole in the structure, and (d) a hole in the structure with distributed loading. All definitions compared can provide identification and visualization of load flow in the structure.

Based on the pre-defined criteria that a load path characterization method should have, namely: (a) visualization of load trajectories, (b) indication for stiffness tailoring, and (c) indication for critical regions, none of the methods compared has clear advantages over the others. Table 1 provides a summary of the methods as they are checked for criteria initially defined.

Table 1. Summary of comparison of various load path definitions against the pre-defined criteria

\begin{tabular}{llll}
\hline Orienta- & Load & Trans- & Load \\
tion of & flow & ferred & paths \\
princip- & based on & and & based \\
al & load & poten- & on $U^{*}$ \\
stresses & equili- & tial & \\
& brium in & trans- & \\
& a set of & ferred & \\
& ortho- & force & \\
& gonal & method & \\
& axes & & \\
\hline
\end{tabular}

Visualiza-

tion of load

$\mathrm{x}$

$\mathrm{X}$

trajectories

Indication

for stiffness

$\mathrm{x}$

tailoring

Indication

for critical $\mathrm{x}$

regions

In several cases and aspects, some methods provide similar results. The examples are:

1. Trajectories from minor principal stress and load flow with equilibrium in $x$-direction under distributed loads.

2. Trajectories from combined principal stresses and load flow with equilibrium in $x$-direction under fixed supports.

3. Regions of high stresses and regions with significant transferred forces.

Further study is needed to have a common approach to characterize and visualize load paths that will meet the predefined criteria.

\section{References}

1. A.P. Boresi, K.P. Chong, Elasticity in engineering mechanics $2^{\text {nd }}$ edition (John Wiley \& Sons, New York, 2000)

2. D.W. Kelly, M.W. Tosh, Interpreting load paths and stress trajectories in elasticity, Engineering Computations, 17(2), 117-135 (2000).

3. D.W. Kelly, P. Hsu, M.W. Tosh, Load paths and load flow in finite element analysis, Engineering Computations, 18(1/2), 304-313 (2001).

4. W. Waldman, M. Heller, R. Kaye, F. Rose, Advances in two-dimensional structural loadflow visualization, Engineering Computations, 19(2), 305-326 (2002).

5. D.W. Kelly, M. Elsley, A procedure for determining load paths in elastic continua, Engineering Computations, 12(5), 415-424 (1995).

6. J.S. Harasaki, J.S. Arora, New concepts of transferred and potential transferred forces in structures, Computer Methods in Applied Mechanics and Engineering, 191(3-5), 385-406 (2001).

7. J.S. Harasaki, J.S. Arora, A new class of evolutionary methods based on the concept of transferred force for structural design, Structural and Multidisciplinary Optimization, 22(1), 35-36 (2001).

8. J.S. Harasaki, J.S. Arora, Topology design based on transferred and potential transferred forces, Structural and Multidisciplinary Optimization, 23(5), 372-381 (2002).

9. H. Hoshino, T. Sakurai, K. Takahashi, Vibration reduction in the cabin of heavy-duty trucks using the theory of load transfer paths, JSAE Review, 24 165-171 (2003).

10. Y. Okano, T. Matsunaga, S. Maruyama, M. Hanazato, K. Takahashi, Load path analysis of vehicle body structures under eigenmode deformation of bending vibration, SAE International, (2009). Available via https://shop.sae.org/technical/papers/2009-01-0770. 